\title{
Distribution des valeurs de transformations méromorphes et applications
}

\author{
Tien-Cuong Dinh et Nessim Sibony
}

\begin{abstract}
A meromorphic transform (MT) between compact Kähler manifolds is a surjective multivalued map with an analytic graph. Let $F_{n}: X \rightarrow X_{n}$ be a sequence of MT. Let $\sigma_{n}$ be an appropriate probability measure on $X_{n}$ and $\sigma$ the product measure of $\sigma_{n}$, on $X:=\prod_{n \geq 1} X_{n}$. We give conditions which imply that$$
\frac{1}{d\left(F_{n}\right)}\left[\left(F_{n}\right)^{*}\left(\delta_{x_{n}}\right)-\left(F_{n}\right)^{*}\left(\delta_{x_{n}^{\prime}}\right)\right] \rightarrow 0
$$

for $\sigma$-almost every $\boldsymbol{x}=\left(x_{1}, x_{2}, \ldots\right)$ and $\boldsymbol{x}^{\prime}=\left(x_{1}^{\prime}, x_{2}^{\prime}, \ldots\right)$ in $\boldsymbol{X}$. Here $\delta_{x_{n}}$ is the Dirac mass at $x_{n}$ and $d\left(F_{n}\right)$ the intermediate degree of maximal order of $F_{n}$.

We introduce a calculus on MT: intermediate degrees of composition and of product of MT. Using this formalism and what we call the $\mathrm{dd}^{\mathrm{c}}$-method, we obtain results on the distribution of common zeros, for random $l$ holomorphic sections of high powers $L^{n}$ of a positive holomorphic line bundle $L$ over a projective manifold.

We also construct the equilibrium measure for random iteration of correspondences. In particular, when $f: X \rightarrow X$ is a meromorphic correspondence of large topological degree $d_{t}$, we show that $d_{t}^{-n}\left(f^{n}\right)^{*} \omega^{k}$ converges to a measure $\mu$, satisfying $f^{*} \mu=d_{t} \mu$. Moreover, quasipsh functions are $\mu$-integrable. Every projective manifold admits such correspondences. When $f$ is a meromorphic map, $\mu$ is exponentially mixing with a precise speed depending on the regularity of the observables.
\end{abstract}

Mathematics Subject Classification (2000). 32H, 37D, 37A25, 81Q50.

Keywords. Meromorphic correspondences, dynamical degrees, speed of mixing, random polynomials.

Notations. Dans tout l'article $(X, \omega),\left(X^{\prime}, \omega^{\prime}\right)$ et $\left(X_{n}, \omega_{n}\right)$ désignent des variétés kählériennes compactes de dimensions respectives $k, k^{\prime}$ et $k_{n}$. On suppose que $\int_{X} \omega^{k}=1, \int_{X^{\prime}} \omega^{\prime k^{\prime}}=1$ et $\int_{X_{n}} \omega_{n}^{k_{n}}=1$ sauf mention contraire. Les constantes géométriques $r(X, \omega), \mathrm{R}_{1}^{*}(X, \omega), \mathrm{R}_{2}^{*}(X, \omega, p)$ sont définies au paragraphe 2.1, $\mathrm{R}_{i}(X, \omega, \sigma), \Delta(X, \omega, \sigma, t)$ au paragraphe 2.2 , les degrés intermédiaires $\lambda_{l}(F)$, les degrés dynamiques $d_{l}(f)$, le degré topologique $d_{t}(f)$ et la constante $\mathrm{A}(f)$ sont définis au paragraphe 3. L'espace projectif complexe $\mathbb{P}^{k}$ est muni de la forme de Fubini- 
Study $\omega_{\mathrm{FS}}$ normalisée par $\int \omega_{\mathrm{FS}}^{k}=1$, de la mesure de probabilité invariante $\Omega_{\mathrm{FS}}$ et d'une autre $m_{\mathrm{FS}}$ sur sa partie réelle $\mathbb{R} \mathbb{P}^{k}$. L'espace multiprojectif $\mathbb{P}^{k, l}=\mathbb{P}^{k} \times \cdots \times \mathbb{P}^{k}$ ( $l$ fois) est muni de la forme de Kähler $\omega_{\mathrm{MP}}$, de la mesure de probabilité invariante naturelle $\Omega_{\mathrm{MP}}$ et d'une autre $m_{\mathrm{MP}}$ sur sa partie réelle (voir l'appendice).

\section{Introduction}

Une variété projective de type général admet au plus un nombre fini d'endomorphismes méromorphes dominants [15]. C'est dire que la dynamique de ces applications est très pauvre. Il n'en est plus de même dès qu'on considère les correspondances. En effet, si $X$ est une variété projective de dimension $k$ et si $g$ et $h$ désignent deux projections holomorphes surjectives de $X$ sur $\mathbb{P}^{k}$, le sous-ensemble analytique

$$
\Gamma:=\{(x, y) \in X \times X, g(x)=h(y)\}
$$

définit une correspondance sur $X$, c.-à-d. une application multivaluée $f:=h^{-1} \circ g$. On peut aussi considérer $h^{-1} \circ u \circ g$, où $u$ est un endomorphisme holomorphe de $\mathbb{P}^{k}$. On obtient ainsi des correspondances dont la dynamique est très riche.

Dans un cadre plus large : celui des transformations méromorphes (TM), bon nombre de questions dynamiques, ou de comportements asymptotiques de préimages, se ramènent à l'étude du problème suivant.

On considère une suite $F_{n}: X \rightarrow X_{n}$ d'applications méromorphes, de correspondances ou plus généralement de TM. On se pose la question de donner des critères vérifiables sur les $F_{n}$ et les $X_{n}$ assurant que les préimages $F_{n}^{-1}\left(x_{n}\right)$ par $F_{n}$ des points $x_{n} \in X_{n}$ sont équidistribuées dans $X$.

Précisons les problèmes. Une TM de codimension $l$ de $X$ dans $X_{n}$ est la donnée d'un sous-ensemble analytique $\Gamma^{(n)}$ de dimension pure $k_{n}+l$ de $X \times X_{n}, 1 \leq l \leq$ $k-1$. On suppose que les projections $\pi$ et $\pi_{n}$, restreintes à chaque composante irréductible de $\Gamma^{(n)}$, sur $X$ et $X_{n}$, sont surjectives. Pour $x_{n} \in X_{n}$ générique, la fibre $F_{n}^{-1}\left(x_{n}\right):=\pi\left(\pi_{n \mid \Gamma^{(n)}}\right)^{-1}\left(x_{n}\right)$ est de dimension $l$. Si $\delta_{x_{n}}$ désigne la masse de Dirac en $x_{n}$, on pose

$$
\left(F_{n}\right)^{*}\left(\delta_{x_{n}}\right):=\pi_{*}\left(\pi_{n \mid \Gamma^{(n)}}\right)^{*} \delta_{x_{n}} .
$$

C'est un courant de bidimension $(l, l)$ porté par $F_{n}^{-1}\left(x_{n}\right)$.

Les graphes $\Gamma^{(n)}$ des $F_{n}$ étant holomorphes, on peut espérer que dans les cas "intéressants", génériquement $F_{n}^{*}\left(\delta_{x_{n}}\right)$ et $F_{n}^{*}\left(\delta_{x_{n}^{\prime}}\right)$, convenablement normalisés, aient la même répartition asymptotique. 
Vol. 81 (2006) Distribution des valeurs de transformations méromorphes et applications 223

Problème d'équidistribution. Trouver des conditions vérifiables, pour que génériquement sur les suites $\left(x_{n}\right)$ et $\left(x_{n}^{\prime}\right)$

$$
\frac{1}{d\left(F_{n}\right)}\left[F_{n}^{*}\left(\delta_{x_{n}}\right)-F_{n}^{*}\left(\delta_{x_{n}^{\prime}}\right)\right] \longrightarrow 0
$$

au sens des courants. Ici $d\left(F_{n}\right)$ désigne la masse d'une fibre générique de $F_{n}$, celle-ci est indépendante de la fibre.

Problème de convergence. Dans certains cas, trouver la limite de la suite de courants

$$
\frac{1}{d\left(F_{n}\right)} F_{n}^{*}\left(\delta_{x_{n}}\right)
$$

Un cas particulier du problème de convergence est celui où on se donne une suite $f_{n}: X_{n-1} \rightarrow X_{n}$ de correspondances, i.e. une suite de TM de codimension 0 entre variétés de même dimension. On veut trouver la limite de la suite de mesures

$$
\frac{1}{d_{1} \ldots d_{n}}\left(f_{n} \circ \cdots \circ f_{1}\right)^{*}\left(\omega_{n}^{k_{n}}\right)
$$

et en donner les propriétés. On est dans le cas où $F_{n}:=f_{n} \circ \cdots \circ f_{1}$, le nombre de points d'une fibre générique de $f_{n}$ est égal à $d_{n}$.

Du point de vue physique, on compose des applications voisines, et un théorème dans ce cadre est signe de la robustesse du résultat. Une seconde raison de considérer des suites d'applications est que pour l'itération d'applications birationnelles $f$, on est amené à considérer le comportement de la suite $\left(f^{n}, f^{-n}\right)$. Nous appliquons ainsi cette approche au problème de la distribution des zéros des sections holomorphes des puissances $L^{n}$ d'un fibré en droites positif $L \rightarrow X$. On prend alors pour $X_{n}$ la projectivisé de l'espace des sections de $L^{n}$. Ce problème est lié au chaos quantique. Voir Nonnenmacher-Voros [19] et Shiffman-Zelditch [22].

Dans le cas général, notre critère d'équidistribution utilise deux notions : l'une liée à la croissance des $F_{n}$ et l'autre à la géométrie des $X_{n}$. Si les $\left(X_{n}, \omega_{n}\right)$ appartiennent à une famille compacte, seule la croissance des $F_{n}$ intervient.

Les indicateurs de croissance sont les degrés intermédiaires d'ordres $k_{n}$ et $k_{n}-1$, classiques en théorie de distribution des valeurs, associés aux $F_{n}$ :

$$
d\left(F_{n}\right):=\int_{X} F_{n}^{*}\left(\omega_{n}^{k_{n}}\right) \wedge \omega^{l} \quad \text { et } \quad \delta\left(F_{n}\right):=\int_{X} F_{n}^{*}\left(\omega_{n}^{k_{n}-1}\right) \wedge \omega^{l+1} .
$$

C'est le comportement de la suite $\delta\left(F_{n}\right) d\left(F_{n}\right)^{-1}$ qui joue un rôle. Le calcul de $d\left(F_{n}\right)$ et $\delta\left(F_{n}\right)$ est cohomologique, $d\left(F_{n}\right)$ est la masse d'une fibre générique de $F_{n}$ et si $X_{n}$ est un espace projectif, $\delta\left(F_{n}\right)$ est la masse de l'image réciproque d'une droite générique. 
La géométrie des variétés intervient par l'intermédiaire des meilleures constantes pour résoudre $\mathrm{dd}^{\mathrm{c}}$ dans une classe de cohomologie donnée. Pour la distribution des zéros des sections aléatoires, la propriété suivante est cruciale : pour $\varphi$ quasi-psh sur $\mathbb{P}^{k}$ avec $\mathrm{dd}^{\mathrm{c}} \varphi \geq-\omega_{\mathrm{FS}}$ et $\int_{\mathbb{P} k} \varphi \mathrm{d} m=0$ on a

$$
\max _{\mathbb{P}^{k}} \varphi \leq c(1+\log k) .
$$

Ici $m$ est la mesure invariante sur $\mathbb{P}^{k}$ ou sur sa partie réelle $\mathbb{R} \mathbb{P}^{k}$ et $c$ est une constante indépendante de $k$. Les estimations des constantes géométriques pour les variétés multiprojectives permettent de déduire la convergence pour les zéros communs de plusieurs sections holomorphes.

Notre approche du problème d'équidistribution général reprend celle que nous avons utilisée pour l'étude des applications à allure polynomiale dans une variété de Stein [5]. Ici, après avoir défini convenablement les images réciproques $F_{n}^{*}$ et les images directes $\left(F_{n}\right)_{*}$ des courants, il faut évaluer

$$
\frac{1}{d\left(F_{n}\right)}\left\langle F_{n}^{*}\left(\delta_{x_{n}}\right)-F_{n}^{*}\left(\omega_{n}^{k_{n}}\right), \psi\right\rangle=\frac{1}{d\left(F_{n}\right)}\left\langle\delta_{x_{n}}-\omega_{n}^{k_{n}},\left(F_{n}\right)_{*} \psi\right\rangle
$$

pour une forme test lisse $\psi$ de bidegré $(l, l)$.

L'idée est de remplacer $\left(F_{n}\right)_{*} \psi$ par une autre solution $\psi_{n}$ de l'équation $\operatorname{dd}^{\mathrm{c}} \psi_{n}=$ $\mathrm{dd}^{\mathrm{c}}\left(F_{n}\right)_{*} \psi$, c.-à-d. de retrancher à $\left(F_{n}\right)_{*} \psi$ une constante convenable. C'est ce que nous appelons la méthode $\mathrm{dd}^{\mathrm{c}}$. Les mesures $\delta_{x_{n}}$ et $\omega_{n}^{k_{n}}$ étant de même masse, le membre à droite de (1.1) ne change pas lorsqu'on remplace $\left(F_{n}\right)_{*} \psi$ par $\psi_{n}$. On estime $\psi_{n}$ en fonction de $\mathrm{dd}^{\mathrm{c}}\left(F_{n}\right)_{*} \psi$, d'où l'introduction de $\delta\left(F_{n}\right)$ qui essentiellement mesure la masse de $\mathrm{dd}^{\mathrm{c}}\left(F_{n}\right)_{*} \psi$.

La vitesse de convergence de $\delta\left(F_{n}\right)\left[d\left(F_{n}\right)\right]^{-1}$ vers 0 a pour conséquence des propriétés de convergence de (1.1) vers 0 sauf sur des ensembles de $\left(x_{n}\right)$ dont nous pouvons majorer la mesure et préciser la nature. Cette méthode $\mathrm{dd}^{\mathrm{c}}$ permet d'obtenir la vitesse de mélange dans le théorème 1.3 ci-dessous ou encore des estimées des volumes de l'ensemble des "mauvaises sections" dans l'étude des zéros de sections aléatoires.

L'article est organisé de la manière suivante. Au paragraphe 2, nous donnons quelques propriétés des fonctions quasi-psh sur une variété kählérienne compacte. On utilise ces fonctions pour tester la convergence. Leurs propriétés de compacité jouent un rôle clé dans les estimations. Nous définissons les opérations image directe et image réciproque des courants que nous utilisons.

Le paragraphe 3 introduit les opérations de composition, de produit et d'intersection, sur les TM. Nous donnons les estimations fondamentales des degrés intermédiaires d'une composée ou d'un produit de TM. C'est un formalisme qui nous l'espérons sera utile dans d'autres questions.

Au paragraphe 4 nous démontrons nos résultats de convergence abstraits sur l'équidistribution, pour des TM générales. Nous les appliquons dans les paragraphes 5, 6, 
Vol. 81 (2006) Distribution des valeurs de transformations méromorphes et applications 225

7 aux diverses situations. Dans un appendice, nous rassemblons les estimations des constantes géométriques qui nous sont nécessaires. Une notion de capacité dans les variétés compactes est également introduite.

Nous précisons maintenant quelques cas simples de nos résultats. Ils seront démontrés aux paragraphes 4 et 5 .

Théorème 1.1. Soient $F_{n}: X \rightarrow X^{\prime}$ des TM de codimension l. Soient $\delta_{n}, d_{n}$ leurs degrés intermédiaires d'ordre $k^{\prime}-1$ et $k^{\prime}$. Notons \& l'ensemble (exceptionnel) des $x^{\prime} \in X$ tels que

$$
\frac{1}{d_{n}}\left[\left(F_{n}\right)^{*}\left(\delta_{x^{\prime}}\right)-\left(F_{n}\right)^{*}\left(\omega^{\prime k^{\prime}}\right)\right] \text { ne tend pas faiblement vers } 0 .
$$

(1) Si $\sum \delta_{n} d_{n}^{-1}<+\infty$ alors \& est pluripolaire.

(2) Si $\sum \exp \left(-\delta_{n}^{-1} d_{n} t\right)<+\infty$ pour tout $t>0$ alors $\mathcal{E}$ est de $\sigma$ mesure nulle pour toute mesure modérée $\sigma$.

Les mesures de Lebesgue sur $X$ ou sur une sous-variété analytique totalement réelle de dimension maximale sont des exemples de mesures modérées (voir paragraphe 2.2). Lorsque les $F_{n}$ sont des applications rationnelles de $\mathbb{P}^{k}$ dans $\mathbb{P}^{k^{\prime}}$ des résultats analogues ont été obtenus par Sodin, Russakovskii et Shiffman [21], [20]. Pour les itérés d'une application holomorphe de $\mathbb{P}^{k}$ voir [10], [23], [2], [5]. Lorsque les $F_{n}$ sont les itérés $f^{n}$ d'une correspondance $f: X \rightarrow X$, le théorème suivant fournit une solution au problème de convergence. C'est un corollaire du théorème 5.1.

Théorème 1.2. Soit $f: X \rightarrow X$ une correspondance méromorphe de degré topologique $d_{t}$. Supposons que son degré dynamique d'ordre $k-1$

$$
d_{k-1}:=\lim _{n \rightarrow \infty}\left(\int_{X}\left(f^{n}\right)^{*} \omega^{k-1} \wedge \omega\right)^{1 / n}
$$

vérifie $d_{k-1}<d_{t}$. Soient $h_{n}$ des fonctions positives vérifiant $\int h_{n} \omega^{k}=1$ et $\lim \sup \left\|h_{n}\right\|_{\mathrm{L}^{2}(X)}^{1 / n}<d_{k-1}^{-1} d_{t}$. Alors $\mu_{n}:=d_{t}^{-n}\left(f^{n}\right)^{*}\left(h_{n} \omega^{k}\right)$ converge vers une mesure de probabilité $\mu$ indépendante de $\left(h_{n}\right)$. De plus, on a $f^{*} \mu=d_{t} \mu$. Si $\varphi$ est une fonction quasi-psh elle est $\mu$-intégrable ( $\mu$ est PB) et $\left\langle\mu_{n}, \varphi\right\rangle \rightarrow\langle\mu, \varphi\rangle$.

Pour montrer que les points périodiques répulsifs sont denses dans le support de $\mu$ et que l'ensemble exceptionnel $\&$ est une réunion finie ou dénombrable d'ensembles analytiques, il suffit d'appliquer directement une méthode géométrique développée par Lyubich [17], Briend-Duval [2] et les auteurs [5], [4]. Rappelons que $\mathcal{E}$ est l'ensemble des points $x$ tels que $d_{t}^{-n}\left(f^{n}\right)^{*} \delta_{x}$ ne converge pas vers $\mu$. Pour toute variété projective $X$ il existe des grandes familles de correspondances vérifiant $d_{k-1}<d_{t}$. 
On montrera que $\mu$ vérifie une propriété de régularité plus forte que PB ( $\mu$ est PC). La notion de mesure PB a été introduite dans [5] et les résultats précédents y sont démontrés dans le cadre des applications d'allure polynomiale. Pour les applications rationnelles sur les variétés projectives, Guedj a, dans un travail récent [12], construit $\mu$ et montré qu'elle est PB. Il a étendu certaines propriétés ci-dessus en utilisant la même méthode géométrique. Dans [6], [7], nous avons montré que $\mu$ est d'entropie maximale $\log d_{t}$.

Théorème 1.3. Soit $f: X \rightarrow X$ une application méromorphe dominante vérifiant $d_{t}>d_{k-1}$. Alors sa mesure d'équilibre $\mu$ est mélangeante avec une vitesse d'ordre $d_{t}^{-n}\left(d_{k-1}+\varepsilon\right)^{n}$ pour tout $\varepsilon>0$. Plus précisément, si $\varphi$ est une fonction de classe $\mathcal{C}^{2}$ et $\psi$ est une fonction bornée, posons

$$
I_{n}(\varphi, \psi):=\int_{X} \varphi\left(\psi \circ f^{n}\right) \mathrm{d} \mu-\left(\int_{X} \varphi \mathrm{d} \mu\right)\left(\int_{X} \psi \mathrm{d} \mu\right) .
$$

Il existe $c>0$ indépendant de $\varphi$ et de $\psi$ tel que

$$
\left|I_{n}(\varphi, \psi)\right| \leq c d_{t}^{-n}\left(d_{k-1}+\varepsilon\right)^{n}\|\varphi\|_{\mathcal{C}^{2}}\|\psi\|_{\mathrm{L}^{\infty}(\mu)}
$$

Ce théorème est en fait valable pour $\varphi$ quasi-psh (voir paragraphe 5). Pour le cas des endomorphismes de $\mathbb{P}^{k}$ voir [10] et pour les applications d'allure polynomiale voir [5]. Si $f: X \rightarrow X$ est une application birationnelle on a $d_{t}=1$ donc les hypothèses des théorèmes 1.2 et 1.3 ne sont pas vérifiées. Nous obtenons cependant au paragraphe 6 un résultat d'équidistribution en considérant simultanément $f^{n}$ et $f^{-n}$. On construit à partir de ces applications des TM naturelles et on applique le théorème 1.1.

Remarques 1.4. Ce texte est une version abrégée d'une prépublication de juin 2003 disponible sur arXiv.org. Certains résultats énoncés alors dans le cas projectif sont étendus ici au cas kählérien grâce au théorème d'approximation des courants démontré par les auteurs dans [7]. Les observables utilisées ici sont les fonctions dsh ou les formes $\mathcal{C}^{2}$. Nous avons noté que les estimations obtenues pour ces observables entraînent des estimations analogues pour les formes ou les fonctions höldériennes (voir aussi [8]). Pour le théorème 1.3, l'interpolation entre les espaces $\mathcal{C}^{0}$ et $\mathcal{C}^{2}$ [25] implique pour tout $\alpha, 0<\alpha \leq 2$, que

$$
\left|I_{n}(\varphi, \psi)\right| \lesssim d_{t}^{-\alpha n / 2}\left(d_{k-1}+\varepsilon\right)^{\alpha n / 2}\|\varphi\|_{\mathcal{C}^{\alpha}}\|\psi\|_{L^{\infty}(\mu)} .
$$

\section{Préliminaires}

Si $S$ est un courant réel fermé de bidegré $(r, r)$ de $X$, notons $\operatorname{cl}(S)$ sa classe dans le groupe de cohomologie

$$
H^{r, r}(X, \mathbb{R}):=H^{r, r}(X, \mathbb{C}) \cap H^{2 r}(X, \mathbb{R}) .
$$


Vol. 81 (2006) Distribution des valeurs de transformations méromorphes et applications 227

On dira que $\operatorname{cl}(S) \leq \operatorname{cl}\left(S^{\prime}\right)$ si $\operatorname{cl}\left(S^{\prime}-S\right)$ peut être représentée par un courant positif fermé. Si $S, S^{\prime}$ sont positifs fermés et $\operatorname{sicl}(S) \leq \operatorname{cl}\left(S^{\prime}\right)$, leurs masses vérifient $\|S\| \leq$ $\left\|S^{\prime}\right\|$, où on a posé $\|S\|:=\int_{X} S \wedge \omega^{k-r}$. Lorsque $S$ est positif fermé, sa masse ne dépend que de $\operatorname{cl}(S)$. Les espaces $\mathrm{L}^{p}(X)$ sont définis par rapport à la forme volume $\omega^{k}$.

2.1. Fonctions quasi-psh et dsh. Une fonction $\varphi: X \rightarrow \mathbb{R} \cup\{-\infty\}$ est quasiplurisousharmonique (quasi-psh) si elle est intégrable, semi-continue supérieurement et vérifie $\operatorname{dd}^{\mathrm{c}} \varphi \geq-c \omega, c>0$, au sens des courants. Une telle fonction appartient à $\mathrm{L}^{p}(X)$ pour tout $p \geq 1$. En effet, localement elle diffère d'une fonction psh par une fonction lisse. Pour toute suite $\left(\varphi_{n}\right)$ de fonctions quasi-psh négatives vérifiant $\mathrm{dd}^{\mathrm{c}} \varphi_{n} \geq-\omega$, on peut extraire une sous-suite qui, ou bien, converge dans tout $\mathrm{L}^{p}(X)$, $p \geq 1$, vers une fonction quasi-psh $\varphi$ vérifiant $\operatorname{dd}^{c} \varphi \geq-\omega$, ou bien, converge uniformément vers $-\infty$, [13, p. 94] (voir aussi [3]). La proposition suivante s'en déduit (voir aussi la proposition 2.3).

Proposition 2.1. La famille des fonctions quasi-psh $\varphi$ vérifiant $\mathrm{dd}^{\mathrm{c}} \varphi \geq-\omega$ et l'une des conditions de normalisation

$$
\max _{X} \varphi=0, \quad \int_{X} \varphi \omega^{k}=0 \quad \text { ou } \quad \int_{X}|\varphi| \omega^{k} \leq A
$$

est compacte dans $\mathrm{L}^{p}(X)$ pour tout $p \geq 1$ où $A>0$ est une constante. De plus, ces fonctions sont bornées supérieurement par une même constante.

Le résultat de compacité précédent est lié à la proposition suivante.

Proposition 2.2. Il existe une constante $r>0$ telle que, pour tout courant positif fermé $T$ de bidegré $(1,1)$ et de masse 1 , il existe une $(1,1)$-forme lisse $\alpha$, qui ne dépend que de $\mathrm{cl}(T)$, et une fonction quasi-psh $\varphi$, vérifiant $-r \omega \leq \alpha \leq r \omega$ et $\operatorname{dd}^{\mathrm{c}} \varphi-T=\alpha$.

On dit qu'un sous-ensemble de $X$ est pluripolaire s'il est contenu dans $(\varphi=-\infty)$ où $\varphi$ est une fonction quasi-psh. On appelle fonction $d s h$ toute fonction, définie hors d'un sous-ensemble pluripolaire, qui s'écrit comme différence de deux fonctions quasi-psh. Deux fonctions dsh sur $X$ sont égales si elles sont égales hors d'un ensemble pluripolaire. Notons $\operatorname{DSH}(X)$ l'espace des fonctions dsh sur $X$. On vérifie facilement que si $\psi$ est dsh sur $X$, il existe deux courants $T^{ \pm}$positifs fermés de bidegré $(1,1)$ tels que $\mathrm{dd}^{\mathrm{c}} \psi=T^{+}-T^{-}$. On a $\operatorname{cl}\left(T^{+}\right)=\operatorname{cl}\left(T^{-}\right)$et $\left\|T^{+}\right\|=\left\|T^{-}\right\|$. Réciproquement, d'après la proposition 2.2 , si $T^{ \pm}$vérifient $\operatorname{cl}\left(T^{+}\right)=\operatorname{cl}\left(T^{-}\right)$, alors $T^{+}-T^{-}=\mathrm{dd}^{\mathrm{c}} \psi$ où $\psi$ est une fonction dsh (on peut choisir $\psi$ telle que $\int_{X} \psi \omega^{k}=0$ ). Posons

$$
\|\psi\|_{\mathrm{DSH}}:=\|\psi\|_{\mathrm{L}^{1}}+\inf \left\{\left\|T^{+}\right\|, \mathrm{dd}^{\mathrm{c}} \psi=T^{+}-T^{-}\right\}
$$


On dit que $\psi_{n}$ converge dans $\operatorname{DSH}(X)$ vers $\psi$ si $\psi_{n} \rightarrow \psi$ au sens des distributions et si $\left(\left\|\psi_{n}\right\|_{\mathrm{DSH}}\right)$ est bornée.

Deux fonctions $\psi_{1}, \psi_{2}$ dans $\mathrm{L}^{1}(X)$ diffèrent par une constante si et seulement si $\mathrm{dd}^{\mathrm{c}} \psi_{1}=\mathrm{dd}^{\mathrm{c}} \psi_{2}$. Nous définissons deux constantes positives liées à la résolution de $\mathrm{dd}^{\mathrm{c}} \operatorname{sur}(X, \omega)$ pour des solutions normalisées. Soit $r(X, \omega)$ la borne inférieure des constantes $r$ qui vérifient la proposition 2.2. Posons

$$
\mathrm{Q}(X, \omega):=\left\{\varphi \text { quasi-psh sur } X, \mathrm{dd}^{\mathrm{c}} \varphi \geq-r(X, \omega) \omega\right\}
$$

et pour tout $p \geq 1$

$$
\begin{aligned}
\mathrm{R}_{1}^{*}(X, \omega) & :=\sup _{\varphi}\left\{\max _{X} \varphi, \varphi \in \mathrm{Q}(X, \omega), \int \varphi \omega^{k}=0\right\} \\
& =\sup _{\varphi}\left\{-\int \varphi \omega^{k}, \varphi \in \mathrm{Q}(X, \omega), \max _{X} \varphi=0\right\}, \\
\mathrm{R}_{2}^{*}(X, \omega, p) & :=\sup _{\varphi}\left\{\|\varphi\|_{\mathrm{L}^{p}(X)}, \varphi \in \mathrm{Q}(X, \omega), \int \varphi \omega^{k}=0\right\} .
\end{aligned}
$$

On verra à la proposition 2.4 , que $\mathrm{R}_{2}^{*}(X, \omega, 1) \leq 2 \mathrm{R}_{1}^{*}(X, \omega)$.

2.2. Mesures PB, PC et mesures modérées. Soit $\mu$ une mesure positive sur $X$. On dira que $\mu$ est $P B$ si les fonctions quasi-psh sont $\mu$-intégrables. Dans le cas de dimension 1, $\mu$ est PB si et seulement si elle admet localement un potentiel borné [5]. Une mesure PB est dite $P C$ si l'application $\psi \mapsto\langle\mu, \psi\rangle$ est continue pour la topologie considérée sur $\operatorname{DSH}(X)$. Soient $c>0$ et $\alpha>0$. Nous dirons qu'une mesure $\mathrm{PB} \mu$ est $(c, \alpha)$-modérée si

$$
\int_{X} \exp (-\alpha \varphi) \mathrm{d} \mu \leq c
$$

pour toute $\varphi$ quasi-psh vérifiant $\mathrm{dd}^{\mathrm{c}} \varphi \geq-\omega$ et $\max _{X} \varphi=0$. On déduit d'un résultat classique [14, p. 105] que la mesure $\omega^{k}$ est $(c, \alpha)$-modérée pour $c$ et $\alpha$ convenables (voir proposition A.2). On verra aussi que les mesures invariantes sur les sous-espaces projectifs réels $\mathbb{R} \mathbb{P}^{k}$ de $\mathbb{P}^{k}$ sont modérées (voir proposition A.7).

Proposition 2.3. Soit $\mu$ une mesure PB sur X. La famille des fonctions quasi-psh $\varphi$, vérifiant $\mathrm{dd}^{\mathrm{c}} \varphi \geq-\omega$, et l'une des conditions de normalisation

$$
\max _{X} \varphi=0, \quad \int_{X} \varphi \mathrm{d} \mu=0 \quad \text { ou } \quad \int_{X}|\varphi| \mu \leq A,
$$

est bornée dans $\mathrm{L}^{1}(\mu)$ et est bornée supérieurement. En particulier, il existe $c>0$ indépendant de $\varphi$ tel que $\mu(\varphi<-t) \leq c t^{-1}$ pour $t>0$. 
Vol. 81 (2006) Distribution des valeurs de transformations méromorphes et applications 229

Démonstration. Soit $\left(\varphi_{n}\right)$ une suite de fonctions quasi-psh vérifiant $\mathrm{dd}^{\mathrm{c}} \varphi_{n} \geq-\omega$. Supposons que $\max _{X} \varphi_{n}=0$. Montrons que $\left(\varphi_{n}\right)$ est bornée dans $\mathrm{L}^{1}(\mu)$. Sinon, quitte à extraire une sous-suite, on peut supposer que $\int \varphi_{n} \mathrm{~d} \mu \leq-n^{2}$. Posons $\Phi:=$ $\sum n^{-2} \varphi_{n}$. D'après la proposition 2.1, $\Phi$ est une fonction quasi-psh vérifiant $\mathrm{dd}^{\mathrm{c}} \Phi \geq$ $-2 \omega$. On a $\int_{X} \Phi \mathrm{d} \mu=-\infty$. Cela contredit que $\Phi$ soit $\mu$-intégrable.

Supposons maintenant que $\int \varphi_{n} \mathrm{~d} \mu=0$. Posons $a_{n}:=\max _{X} \varphi_{n}$ et $\widetilde{\varphi}_{n}:=\varphi_{n}-a_{n}$. On a $\max _{X} \widetilde{\varphi}_{n}=0$. D'après la partie précédente, $\left(\widetilde{\varphi}_{n}\right)$ est bornée dans $\mathrm{L}^{1}(\mu)$. Or $a_{n}=-\int \widetilde{\varphi}_{n} \mathrm{~d} \mu$, donc $\left(a_{n}\right)$ est bornée et par suite $\left(\varphi_{n}\right)$ est bornée dans $\mathrm{L}^{1}(\mu)$. On en déduit aussi que $\left(\varphi_{n}\right)$ est bornée supérieurement. Le troisième cas se traite de façon similaire. Si $c$ est tel que $\|\varphi\|_{\mathrm{L}^{1}(\mu)} \leq c$, on a $\mu(\varphi<-t) \leq c t^{-1}$.

Soit $\mu$ une mesure de probabilité PB. Il résulte de la proposition 2.3 qu'on peut

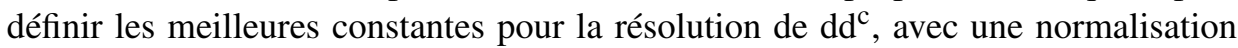
associée à $\mu$. Posons

$$
\begin{aligned}
\mathrm{R}_{1}(X, \omega, \mu) & :=\sup _{\varphi}\left\{\max _{X} \varphi, \varphi \in \mathrm{Q}(X, \omega), \int \varphi \mathrm{d} \mu=0\right\} \\
& =\sup _{\varphi}\left\{-\int \varphi \mathrm{d} \mu, \varphi \in \mathrm{Q}(X, \omega), \max _{X} \varphi=0\right\}, \\
\mathrm{R}_{2}(X, \omega, \mu) & :=\sup _{\varphi}\left\{\|\varphi\|_{\mathrm{L}^{1}(\mu)}, \varphi \in \mathrm{Q}(X, \omega), \int \varphi \mathrm{d} \mu=0\right\}, \\
\mathrm{R}_{3}(X, \omega, \mu) & :=\sup _{\varphi}\left\{\left|\int \varphi \omega^{k}\right|, \varphi \in \mathrm{Q}(X, \omega), \int \varphi \mathrm{d} \mu=0\right\} \\
& :=\sup _{\varphi}\left\{\left|\int \varphi \mathrm{d} \mu\right|, \varphi \in \mathrm{Q}(X, \omega), \int \varphi \omega^{k}=0\right\},
\end{aligned}
$$

et pour tout $t \in \mathbb{R}$

$$
\Delta(X, \omega, \mu, t):=\sup _{\varphi}\left\{\mu(\varphi<-t), \varphi \in \mathrm{Q}(X, \omega), \int \varphi \mathrm{d} \mu=0\right\} .
$$

\section{Proposition 2.4. On $a$}

$$
\mathrm{R}_{2}(X, \omega, \mu) \leq 2 \mathrm{R}_{1}(X, \omega, \mu) \quad \text { et } \quad \mathrm{R}_{3}(X, \omega, \mu) \leq \mathrm{R}_{1}(X, \omega, \mu)+\mathrm{R}_{1}^{*}(X, \omega) .
$$

Si $\mu$ est $(c, \alpha)$-modérée alors $\Delta(X, \omega, \mu, t) \leq c \exp \left(-\alpha r^{-1} t\right)$ où $r:=r(X, \omega)$.

Démonstration. Soit $\varphi$ une fonction quasi-psh telle que $\mathrm{dd}^{\mathrm{c}} \varphi \geq-r \omega$ et $\int \varphi \mathrm{d} \mu=0$. Posons $m:=\max _{X} \varphi$. On $\operatorname{aax}_{X}(\varphi-m)=0$, donc

$$
\int|\varphi| \mathrm{d} \mu \leq \int|\varphi-m| \mathrm{d} \mu+m=\int(m-\varphi) \mathrm{d} \mu+m=2 m \leq 2 \mathrm{R}_{1}(X, \omega, \mu) .
$$


D'où $\mathrm{R}_{2}(X, \omega, \mu) \leq 2 \mathrm{R}_{1}(X, \omega, \mu)$. D'après (2.2), on a

$$
\begin{aligned}
\left|\int \varphi \omega^{k}\right| & \leq \int|\varphi-m| \omega^{k}+m=\int(m-\varphi) \omega^{k}+m \\
& \leq \mathrm{R}_{1}^{*}(X, \omega)+\mathrm{R}_{1}(X, \omega, \mu) .
\end{aligned}
$$

Ceci implique que $\mathrm{R}_{3}(X, \omega, \mu) \leq \mathrm{R}_{1}(X, \omega, \mu)+\mathrm{R}_{1}^{*}(X, \omega)$.

La fonction $\psi:=r^{-1}(\varphi-m)$ vérifie $\mathrm{dd}^{\mathrm{c}} \psi \geq-\omega$ et $\max _{X} \psi=0$. Si $\mu$ est $(c, \alpha)$-modérée, on a $\int \exp (-\alpha \psi) \mathrm{d} \mu \leq c$. D'autre part, comme $\int \varphi \mathrm{d} \mu=0$, on a $m \geq 0$, donc $\psi \leq r^{-1} \varphi$. On en déduit que

$$
\mu(\varphi<-t) \leq \mu\left(\psi<-r^{-1} t\right) \leq c \exp \left(-\alpha r^{-1} t\right) .
$$

Ceci implique que $\Delta(X, \omega, \mu, t) \leq c \exp \left(-\alpha r^{-1} t\right)$.

2.3. Image directe d'un courant. Soit $\pi: X \rightarrow X^{\prime}$ une application holomorphe surjective. Si $S$ est un courant de bidimension $(r, r)$ de $X$, avec $0 \leq r \leq \min \left(k, k^{\prime}\right)$, le courant $\pi_{*}(S)$ est défini par

$$
\left\langle\pi_{*}(S), \psi\right\rangle:=\left\langle S, \pi^{*}(\psi)\right\rangle
$$

pour toute $(r, r)$-forme lisse $\psi$ sur $X^{\prime}$. Si $S$ est une forme à coefficients dans $\mathrm{L}^{1}, \pi_{*}(S)$ l'est aussi. Les coefficients de $\pi_{*}(S)$ sont obtenus par intégration sur les fibres qui sont presque partout de même dimension.

Proposition 2.5. Si les fibres génériques de $\pi$ sont finies, l'application $\pi_{*}: \mathrm{DSH}(X) \rightarrow \mathrm{DSH}\left(X^{\prime}\right)$ est bien définie, bornée et continue. En particulier, l'image de $\left\{\varphi\right.$ quasi-psh sur $\left.X, \operatorname{dd}^{\mathrm{c}} \varphi \geq-\omega, \int_{X} \varphi \omega^{k}=0\right\}$ par $\pi_{*}$ est relativement compacte dans $\mathrm{L}^{p}\left(X^{\prime}\right)$ pour tout $p \geq 1$.

Démonstration. Notons $I(\pi)$ l'ensemble des $x^{\prime} \in X^{\prime}$ tels que $\pi^{-1}\left(x^{\prime}\right)$ ne soit pas finie. La fonction $\pi_{*}(\psi)$ est définie hors de l'ensemble $I(\pi)$ qui est fermé et pluripolaire (voir proposition A.1). Pour $x^{\prime} \in X^{\prime} \backslash I(\pi)$ on a

$$
\pi_{*}(\psi)\left(x^{\prime}\right)=\sum_{\pi\left(x_{i}\right)=x^{\prime}} \psi\left(x_{i}\right) .
$$

Soient $T^{ \pm}$des courants positifs fermés tels que $\mathrm{dd}^{\mathrm{c}} \psi=T^{+}-T^{-}$. On a dd ${ }^{\mathrm{c}} \pi_{*} \psi=$ $\pi_{*}\left(T^{+}\right)-\pi_{*}\left(T^{-}\right)$(voir aussi [18]). Puisque les courants $\pi_{*}\left(T^{ \pm}\right)$sont positifs fermés, $\pi_{*}(\psi)$ est dsh. Observons que l'opérateur $\pi_{*}: \mathrm{L}^{1}(X) \rightarrow \mathrm{L}^{1}\left(X^{\prime}\right)$ est borné et que $\left\|\pi_{*}\left(T^{ \pm}\right)\right\| \leq c\left\|T^{ \pm}\right\|$avec $c>0$ indépendant de $T^{ \pm}$. On en déduit que $\pi_{*}: \operatorname{DSH}(X) \rightarrow \operatorname{DSH}\left(X^{\prime}\right)$ est borné et continu pour la topologie considérée sur les fonctions dsh. 
Vol. 81 (2006) Distribution des valeurs de transformations méromorphes et applications 231

2.4. Image réciproque d'un courant. Soit $\pi: X \rightarrow X^{\prime}$ une application holomorphe surjective. L'image réciproque $\pi^{*}(\alpha)$ d'un courant $\alpha$ sur $X^{\prime}$ est définie lorsque $\pi$ est une submersion. En général, on peut définir $\pi^{*}$ dans les cas suivants. Lorsque $\gamma$ est une $(p, q)$-forme à coefficients dans $\mathrm{L}^{\infty}\left(X^{\prime}\right)$, la forme $\pi^{*}(\gamma)$ est à coefficients dans $\mathrm{L}^{\infty}(X)$. Lorsque $\psi$ est une fonction dsh sur $X^{\prime}, \psi \circ \pi$ est aussi une fonction dsh sur $X$. On peut définir $\pi^{*}(\psi \gamma):=(\psi \circ \pi) \pi^{*}(\gamma)$. On vérifie facilement le résultat suivant.

Proposition 2.6. L'opérateur $\pi^{*}: \operatorname{DSH}\left(X^{\prime}\right) \rightarrow \operatorname{DSH}(X)$ est borné. En particulier, l'image de $\left\{\varphi\right.$ quasi-psh sur $\left.X^{\prime}, \operatorname{dd}^{\mathrm{c}} \varphi \geq-\omega^{\prime}, \int_{X^{\prime}} \varphi \omega^{\prime k^{\prime}}=0\right\}$ par $\pi^{*}$ est relativement compacte dans $\mathrm{L}^{p}(X)$ pour tout $p \geq 1$.

Rappelons la définition de $\pi^{*}$ sur les courants positifs fermés de bidegré $(1,1)$. Soit $T$ un tel courant sur $X^{\prime}$. Il existe une fonction quasi-psh $\psi$ sur $X^{\prime}$ et une forme lisse $\alpha$ telles que $\mathrm{dd}^{\mathrm{c}} \psi=T-\alpha$. On pose $\varphi:=\psi \circ \pi$ et $\pi^{*}(T):=\operatorname{dd}^{\mathrm{c}} \varphi+\pi^{*}(\alpha)$. C'est un courant positif fermé de masse finie. Cette opération est continue et indépendante du choix de $\alpha$ et de $\psi$ [18]. On a $\operatorname{cl}\left(\pi^{*}(T)\right)=\operatorname{cl}\left(\pi^{*}(\alpha)\right)$. Si $\psi$ est une fonction dsh sur $X^{\prime}$ avec $\mathrm{dd}^{\mathrm{c}} \psi=T^{+}-T^{-}$, on a dd $\pi^{*}(\psi)=\pi^{*}\left(T^{+}\right)-\pi^{*}\left(T^{-}\right)$.

Notons $I$ l'ensemble des $x^{\prime} \in X^{\prime}$ tels que $\operatorname{dim} \pi^{-1}\left(x^{\prime}\right)>k-k^{\prime}$. C'est un sous-ensemble analytique de codimension au moins 2 de $X^{\prime}$ car $\operatorname{dim} \pi^{-1}(I)$ est au plus égale à $k-1$. Soit $H$ un sous-ensemble analytique de dimension pure $l$ de $X^{\prime}$. Supposons que $\operatorname{dim} \pi^{-1}(H \cap I)<l+k-k^{\prime}$. On définit $\pi^{*}[H]$ comme un courant d'intégration sur $\pi^{-1}(H)$, il est alors de même bidegré que $[H]$.

Si $T$ est un courant positif fermé de bidegré $(r, r)$ sur $X^{\prime}$, on peut définir "la partie principale" de l'image réciproque de $T$. Soit $\Omega \subset X$ l'ouvert, Zariski dense, où $\pi$ est une submersion locale. Le courant $\left(\pi_{\mid \Omega}\right)^{*}(T)$ est bien défini, positif, fermé sur $\Omega$. Notons $\widetilde{(\pi \mid \Omega)^{*}(T)}$ son prolongement trivial.

Proposition 2.7 ([6], [7]). Le courant $\left(\pi_{\mid \Omega}\right)^{*}(T)$ est de masse finie et le courant $(\widetilde{\pi \mid \Omega})^{*}(T)$ est fermé. Si $T_{n} \rightarrow T$, tout courant adhérent à la suite $\left(\widetilde{\left.\pi_{\mid \Omega}\right)^{*} T_{n}}\right.$ est supérieur ou égal à $\widetilde{(\pi \mid \Omega) * T}$.

\section{Transformations méromorphes}

Dans ce paragraphe, nous définissons les opérations : composition, produit, intersection, sur les TM et les correspondances méromorphes. Nous étudions l'effet de ces opérations sur les degrés intermédiaires.

3.1. Définitions. Notons $\pi_{i}: X_{1} \times X_{2} \rightarrow X_{i}$ la projection canonique de $X_{1} \times X_{2}$ sur $X_{i}$. On appelle $m$-chaîne holomorphe (positive) de $X_{1} \times X_{2}$ toute combinaison 
finie $\Gamma:=\sum \Gamma_{j}$ où les $\Gamma_{j}$ sont des sous-ensembles analytiques irréductibles de dimension $m$ de $X_{1} \times X_{2}$. Les $\Gamma_{j}$ ne sont pas nécessairement distincts. L'intégration sur la partie lisse de $\Gamma$ définit un courant positif fermé $[\Gamma]$ de bidimension $(m, m)$. Notons $\bar{\Gamma} \subset X_{2} \times X_{1}$ l'image de $\Gamma$ par l'application $\left(x_{1}, x_{2}\right) \mapsto\left(x_{2}, x_{1}\right)$.

Soit $l$ un entier naturel, $k_{1}-k_{2} \leq l<k_{1}$. On appelle transformation méromorphe (TM) $F$ de $X_{1}$ dans $X_{2}$ toute $\left(k_{2}+l\right)$-chaîne holomorphe $\Gamma=\sum \Gamma_{j}$ de $X_{1} \times X_{2}$ telle que la restriction de $\pi_{i}$ à chaque composante irréductible $\Gamma_{j}$ soit surjective, $i=1,2$. On dira que $\Gamma$ est le graphe de $F$ et que $\operatorname{codim}(F):=l$ est la codimension de $F$. La TM $\bar{F}$ de $X_{2}$ dans $X_{1}$ associée à $\bar{\Gamma}$ est appelée TM adjointe de $F$, elle est de codimension $k_{2}-k_{1}+l$. Posons $F:=\pi_{2} \circ\left(\pi_{1 \mid \Gamma}\right)^{-1}$ et $F^{-1}:=\pi_{1} \circ\left(\pi_{2 \mid \Gamma}\right)^{-1}$. Ces "applications" sont définies sur les sous-ensembles de $X_{1}$ et $X_{2}$. La fibre $F^{-1}\left(x_{2}\right)$ de $x_{2} \in X_{2}$ est génériquement un sous-ensemble analytique de dimension $l$ de $X_{1}$. Notons $I_{i}(F):=\left\{x \in X_{i}, \operatorname{dim} \pi_{i}^{-1}(x)>k_{2}+l-k_{i}\right\}$. C'est un sous-ensemble analytique de codimension au moins 2 de $X_{i}$. On dira que $I_{1}(F)\left(\right.$ resp. $\left.I_{2}(F)\right)$ est le premier (resp. deuxième) ensemble d'indétermination.

Définissons les opérateurs $F^{*}$ et $F_{*}$. Soit $T$ un courant de bidegré $(r, r)$ sur $X_{2}$, $k_{2}+l-k_{1} \leq r \leq k_{2}$. On définit $F^{*}(T):=\left(\pi_{1}\right)_{*}\left(\pi_{2}^{*}(T) \wedge[\Gamma]\right)$. C'est un courant de bidimension $\left(k_{2}+l-r, k_{2}+l-r\right)$ porté par $F^{-1}(\operatorname{supp}(T))$. Cet opérateur est défini pour les formes lisses et pour l'espace engendré par les courants [H] où $H$ est un sous-ensemble analytique de dimension pure $k_{2}-r$ de $X_{2}$ vérifiant $\operatorname{dim}\left(\pi_{2 \mid \Gamma}\right)^{-1}(H \cap$ $\left.I_{2}(F)\right) \leq k_{2}+l-r-1$. Lorsque $T$ est lisse, $F^{*}(T)$ est une forme à coefficients dans $\mathrm{L}^{1}(X)$. Pour $x_{2} \in X_{2} \backslash I_{2}(F), F^{*}\left(\delta_{x_{2}}\right)$ est un courant d'intégration sur une $l$-chaîne holomorphe portée par $F^{-1}\left(x_{2}\right)$. L'opérateur $F_{*}$ est défini de la même manière.

Pour tout $s, k_{2}-k_{1}+l \leq s \leq k_{2}$, on appelle degré intermédiaire d'ordre $s$ de $F$ le nombre

$$
\begin{aligned}
\lambda_{s}(F) & :=\int_{X_{1}} F^{*}\left(\omega_{2}^{s}\right) \wedge \omega_{1}^{k_{2}+l-s}=\int_{X_{2}} \omega_{2}^{s} \wedge F_{*}\left(\omega_{1}^{k_{2}+l-s}\right) \\
& =\int_{\Gamma} \pi_{1}^{*}\left(\omega_{1}^{k_{2}+l-s}\right) \wedge \pi_{2}^{*}\left(\omega_{2}^{s}\right) .
\end{aligned}
$$

Par continuité, la masse du courant $F^{*}\left(\delta_{x_{2}}\right)$, qui se calcule cohomologiquement, ne dépend pas de $x_{2}$, pour $x_{2}$ générique dans $X_{2}$. On en déduit que cette masse est égale au dernier degré intermédiaire $\lambda_{k_{2}}(F)$ de $F$.

Finalement, on dira qu'un point $\left(x_{1}, x_{2}\right) \in \Gamma$ est générique si la restriction de $\pi_{i \mid \Gamma}$ à un voisinage de $\left(x_{1}, x_{2}\right)$ est une submersion pour $i=1,2$. Notons $\operatorname{Gen}(\Gamma)$ l'ensemble de ces points. C'est un ouvert de Zariski dense de $\Gamma$.

3.2. Composition de TM. Soit $F^{\prime}: X_{2} \rightarrow X_{3}$ une autre TM, de codimension $l^{\prime}$, associée à une $\left(k_{3}+l^{\prime}\right)$-chaîne holomorphe $\Gamma^{\prime}=\sum \Gamma_{j}^{\prime}$ de $X_{2} \times X_{3}$. Supposons que $l+l^{\prime}<k_{1}$. Considérons d'abord le cas où $\Gamma$ et $\Gamma^{\prime}$ sont irréductibles. Définissons la composée $\Gamma^{\prime} \circ \Gamma$ des graphes. Notons $\pi_{1}, \pi_{2}$ les projections de $X_{1} \times X_{2}$ sur $X_{1}$ et 
Vol. 81 (2006) Distribution des valeurs de transformations méromorphes et applications 233

$X_{2}$ et $\pi_{2}^{\prime}, \pi_{3}^{\prime}$ les projections de $X_{2} \times X_{3}$ sur $X_{2}$ et $X_{3}$. Soient $\left(x_{1}, x_{2}\right) \in \operatorname{Gen}(\Gamma)$ et $\left(x_{2}, x_{3}\right) \in \operatorname{Gen}\left(\Gamma^{\prime}\right)$. Soient $U \subset \operatorname{Gen}(\Gamma)$ et $U^{\prime} \subset \operatorname{Gen}\left(\Gamma^{\prime}\right)$ des petits voisinages de $\left(x_{1}, x_{2}\right)$ dans $\operatorname{Gen}(\Gamma)$ et de $\left(x_{2}, x_{3}\right)$ dans $\operatorname{Gen}\left(\Gamma^{\prime}\right)$. Par définition de $\operatorname{Gen}(\Gamma)$ et $\operatorname{Gen}\left(\Gamma^{\prime}\right)$, on peut supposer que $U$ et $U^{\prime}$ admettent des structures produit $U \simeq W_{1} \times V_{2}$ et $U^{\prime} \simeq V_{2} \times W_{3}$ où $V_{2}$ désigne un voisinage de $x_{2}$ dans $X_{2}$. Les projections $\pi_{2}, \pi_{2}^{\prime}$ de $U$ et $U^{\prime}$ sur $X_{2}$ coïncident avec les projections des produits sur le facteur $V_{2}$. Les projections de $U$ sur $X_{1}$ et de $U^{\prime}$ sur $X_{3}$ correspondent à des applications holomorphes $\tau: U \rightarrow X_{1}$ et $\tau^{\prime}: U^{\prime} \rightarrow X_{3}$.

Le modèle local de $\Gamma^{\prime} \circ \Gamma$ est l'image de $W_{1} \times V_{2} \times W_{3}$ dans $X_{1} \times X_{3}$ par l'application $\left(x_{1}, x_{2}, x_{3}\right) \mapsto\left(\tau\left(x_{1}, x_{2}\right), \tau^{\prime}\left(x_{2}, x_{3}\right)\right)$. Cette image est de dimension $\leq k_{3}+l+l^{\prime}$. On suppose qu'elle est de dimension $k_{3}+l+l^{\prime}$. On dira alors que $\Gamma$ et $\Gamma^{\prime}$ se composent correctement. Le graphe $\Gamma^{\prime} \circ \Gamma$ de $F^{\prime} \circ F$ est alors l'adhérence de l'ensemble des $\left(x_{1}, x_{3}\right) \in X_{1} \times X_{3}$ pour lesquels il existe $x_{2} \in X_{2}$ avec $\left(x_{1}, x_{2}\right) \in \operatorname{Gen}(\Gamma)$ et $\left(x_{2}, x_{3}\right) \in \operatorname{Gen}\left(\Gamma^{\prime}\right)$ tel qu'aux voisinages de ces points $\Gamma$ et $\Gamma^{\prime}$ se composent correctement. Le point $\left(x_{1}, x_{3}\right)$ est compté avec la multiplicité $m$ si $m$ est le nombre des points $x_{2}$ pour lesquels $x_{1}, x_{2}, x_{3}$ vérifient la propriété ci-dessus. Puisque $\Gamma$ et $\Gamma^{\prime}$ se composent correctement, $m$ est fini.

Dans le cas où $\Gamma$ et $\Gamma^{\prime}$ ne sont pas irréductibles, on pose $\Gamma^{\prime} \circ \Gamma:=\sum \Gamma_{j}^{\prime} \circ \Gamma_{i}$ en supposant que $\Gamma_{i}$ et $\Gamma_{j}^{\prime}$ se composent correctement pour tout $i, j$. Observons que $\Gamma^{\prime} \circ \Gamma$ est une $\left(k_{3}+l+l^{\prime}\right)$-chaîne holomorphe et qu'on a $\operatorname{codim}\left(F^{\prime} \circ F\right)=$ $\operatorname{codim}(F)+\operatorname{codim}\left(F^{\prime}\right)=l+l^{\prime}$. Les TM de codimension 0 entre variétés de même dimension (c.-à-d. les correspondances) se composent toujours correctement.

La composition $\Gamma^{\prime} \circ \Gamma$ peut se définir de la manière suivante. $\mathrm{Si}\left(x_{1}, x_{2}, x_{2}^{\prime}, x_{3}\right)$ désigne les coordonnées de $X_{1} \times X_{2} \times X_{2} \times X_{3}$, on note $\widetilde{\Gamma}$ l'adhérence de $\operatorname{Gen}(\Gamma) \times \operatorname{Gen}\left(\Gamma^{\prime}\right) \cap\left(x_{2}=x_{2}^{\prime}\right)$. On obtient $\Gamma^{\prime} \circ \Gamma$ comme la projection de $\widetilde{\Gamma}$ sur $X_{1} \times X_{3}$. L'hypothèse qu'on a posée dit que cette projection est de dimension pure $k_{2}+l+l^{\prime}$. Sans cette hypothèse on peut aussi définir la composition en supprimant les composantes de mauvaise dimension.

Proposition 3.1. Il existe une constante $c>0$, qui ne dépend que de $\left(X_{2}, \omega_{2}\right)$, telle que pour tout $s, k_{3}-k_{1}+l+l^{\prime} \leq s \leq k_{3}$, on ait

$$
\lambda_{s}\left(F^{\prime} \circ F\right) \leq c \lambda_{k_{2}-k_{3}+s-l^{\prime}}(F) \lambda_{s}\left(F^{\prime}\right) .
$$

Démonstration. Observons que dans (3.1) les formes étant lisses ou à coefficients dans $\mathrm{L}^{1}$, les intégrales peuvent ne porter que sur des ouverts de mesure totale. Posons $S:=\left(F^{\prime}\right)^{*}\left(\omega_{3}^{s}\right)$. C'est un courant positif fermé à coefficients dans $\mathrm{L}^{1}\left(X_{2}\right)$ de bidegré $(r, r)$ sur $X_{2}$ où $r:=k_{2}-k_{3}+s-l^{\prime}$. On a $\|S\|=\lambda_{s}\left(F^{\prime}\right)$. D'après [7], il existe $c>0$ et des courants $S_{m}$ positifs fermés lisses cohomologiquement dominés par $c\|S\| \omega_{2}^{r}$ 
qui convergent vers un courant $S^{\prime}$ vérifiant $S^{\prime} \geq S$. On a

$$
\begin{aligned}
\lambda_{s}\left(F^{\prime} \circ F\right) & =\int_{\operatorname{Gen}(\Gamma)}\left(\pi_{1}\right)^{*}\left(\omega_{1}^{k_{3}+l+l^{\prime}-s}\right) \wedge\left(\pi_{2}\right)^{*}(S) \\
& \leq \int_{\operatorname{Gen}(\Gamma)}\left(\pi_{1}\right)^{*}\left(\omega_{1}^{k_{3}+l+l^{\prime}-s}\right) \wedge\left(\pi_{2}\right)^{*}\left(S^{\prime}\right) \\
& \leq \lim _{m \rightarrow \infty} \int_{\Gamma}\left(\pi_{1}\right)^{*}\left(\omega_{1}^{k_{3}+l+l^{\prime}-s}\right) \wedge\left(\pi_{2}\right)^{*}\left(S_{m}\right) \\
& \leq c \lambda_{s}\left(F^{\prime}\right) \int_{\Gamma}\left(\pi_{1}\right)^{*}\left(\omega_{1}^{k_{3}+l+l^{\prime}-s}\right) \wedge\left(\pi_{2}\right)^{*}\left(\omega^{r}\right) \\
& =c \lambda_{k_{2}-k_{3}+s-l^{\prime}}(F) \lambda_{s}\left(F^{\prime}\right) .
\end{aligned}
$$

La première égalité résulte de la description locale de $\Gamma^{\prime} \circ \Gamma$, la linéarité permet ensuite d'utiliser des partitions de l'unité. Pour la deuxième inégalité, on utilise une suite exhaustive de compacts de $\operatorname{Gen}(\Gamma)$. On peut aussi démontrer cette proposition en utilisant l'idée de la proposition 3.2 qui suit.

3.3. Produit et intersection de TM. Considérons deux TM $F_{i}: X \rightarrow X_{i}$ de codimensions $l_{i}, i=1,2$. On suppose que $l_{1}+l_{2} \geq k$ et qu'il existe un ouvert, Zariski dense, $\Omega \subset X_{1} \times X_{2}$ tel que $F_{1}^{-1}\left(x_{1}\right) \cap F_{2}^{-1}\left(x_{2}\right)$ soit de dimension pure $l_{1}+l_{2}-k$ pour tout $\left(x_{1}, x_{2}\right) \in \Omega$. On suppose aussi pour simplifier que cette intersection est transverse en tout point générique mais cette hypothèse n'est pas indispensable.

Définissons le produit $F_{1} \bullet F_{2}$. C'est une TM de $X$ dans $X_{1} \times X_{2}$ de codimension $l_{1}+l_{2}-k$ dont nous allons décrire le graphe. Notons $\Gamma^{1}=\sum \Gamma_{i}^{1}$ et $\Gamma^{2}=\sum \Gamma_{j}^{2}$ les graphes de $F_{1}$ et $F_{2}$. Considérons d'abord le cas où $\Gamma^{1}$ et $\Gamma^{2}$ sont irréductibles. Le graphe $\Gamma^{1} \bullet \Gamma^{2}$ de $F_{1} \bullet F_{2}$ est alors l'adhérence de l'ensemble des $\left(x, x_{1}, x_{2}\right) \in X \times \Omega$ avec $x \in F_{1}^{-1}\left(x_{1}\right) \cap F_{2}^{-1}\left(x_{2}\right)$. Dans le cas général, on pose $\Gamma^{1} \bullet \Gamma^{2}:=\sum \Gamma_{i}^{1} \bullet \Gamma_{j}^{2}$.

On peut construire $\Gamma^{1} \bullet \Gamma^{2}$ autrement. Soient $\left(x, x_{1}, x^{\prime}, x_{2}\right)$ les coordonnées de $X \times X_{1} \times X \times X_{2}$. On note $\widetilde{\Omega}:=\left\{\left(x, x_{1}, x^{\prime}, x_{2}\right),\left(x_{1}, x_{2}\right) \in \Omega\right\}$ et $\widetilde{\Gamma}$ l'adhérence de $\left(\Gamma^{1} \times \Gamma^{2}\right) \cap\left(x=x^{\prime}\right) \cap \widetilde{\Omega}$. Alors $\Gamma^{1} \bullet \Gamma^{2}$ est la projection de $\widetilde{\Gamma}$ sur $X \times X_{1} \times X_{2}$.

On munit $X_{1} \times X_{2}$ de la forme de Kähler $\omega_{12}:=c_{12}\left(\pi_{1}^{*}\left(\omega_{1}\right)+\pi_{2}^{*}\left(\omega_{2}\right)\right)$ où $\pi_{i}$ est la projection canonique sur $X_{i}$ et $c_{12}^{-k_{1}-k_{2}}:=\left(\begin{array}{c}k_{1}+k_{2} \\ k_{1}\end{array}\right)$. Le choix de $c_{12}$ implique que $\int_{X_{1} \times X_{2}}\left(\omega_{12}\right)^{k_{1}+k_{2}}=1$.

Proposition 3.2. Il existe une constante $c>0$ qui ne dépend que de $(X, \omega)$ telle que pour tout $s$ vérifiant $k_{1}+k_{2}-2 k+l_{1}+l_{2} \leq s \leq k_{1}+k_{2}$ on ait

$$
\lambda_{s}\left(F_{1} \bullet F_{2}\right) \leq c c_{12}^{s} \sum \lambda_{s_{1}}\left(F_{1}\right) \lambda_{s_{2}}\left(F_{2}\right)
$$

avec $k_{1}-k+l_{1} \leq s_{1} \leq k_{1}, k_{2}-k+l_{2} \leq s_{2} \leq k_{2}$ et $s_{1}+s_{2}=s$. 
Vol. 81 (2006) Distribution des valeurs de transformations méromorphes et applications 235

Démonstration. Soient $\Pi, \Pi_{1}, \Pi^{\prime}, \Pi_{2}$ les projections de $X \times X_{1} \times X \times X_{2}$ sur les facteurs et $\Psi:=\left(\Pi, \Pi^{\prime}\right)$. Soit $r:=k_{1}+k_{2}+l_{1}+l_{2}-k-s$. Alors $c_{12}^{-s} \lambda_{s}\left(F_{1} \bullet F_{2}\right)$ est la masse de la mesure $[\widetilde{\Gamma}] \wedge \Pi^{*}\left(\omega^{r}\right) \wedge\left(\Pi_{1}^{*}\left(\omega_{1}\right)+\left(\Pi_{2}\right)^{*}\left(\omega_{2}\right)\right)^{s}$. Soit $\Delta$ la diagonale de $X \times X$. Par hypothèse, $\Psi$ restreinte à $\Gamma^{1} \times \Gamma^{2}$ est une submersion en un point générique de $\left(\Gamma^{1} \times \Gamma^{2}\right) \cap \Psi^{-1}(\Delta)$. On peut trouver un ouvert de Zariski $\widehat{\Gamma}$ de $\Gamma^{1} \times \Gamma^{2}$ sur lequel la mesure considérée est de masse totale et $\Psi_{\mid \widehat{\Gamma}}$ est une submersion. On a $[\widetilde{\Gamma}]=[\widehat{\Gamma}] \wedge \Psi^{*}[\Delta]$. D'après [7], il existe des courants lisses $\Delta_{m}$ cohomologiquement dominés par $c_{X}\left(\omega(x)+\omega\left(x^{\prime}\right)\right)^{k}, c_{X}>0$, qui tendent vers un courant $\Delta^{\prime} \geq[\Delta]$. Comme dans la proposition 3.1, on montre que la masse de la mesure considérée est majorée par

$$
c_{X} \int\left[\Gamma^{1} \times \Gamma^{2}\right] \wedge\left(\Pi^{*}(\omega)+\Pi^{\prime *}(\omega)\right)^{k} \wedge \Pi^{*}\left(\omega^{r}\right) \wedge\left(\Pi_{1}^{*}\left(\omega_{1}\right)+\left(\Pi_{2}\right)^{*}\left(\omega_{2}\right)\right)^{s} .
$$

La dernière intégrale est une combinaison d'au plus $k$ termes du type

$$
\left(\int\left[\Gamma^{1}\right] \wedge \pi^{*}(\omega)^{k_{1}+l_{1}-s_{1}} \wedge \pi_{1}^{*}\left(\omega_{1}\right)^{s_{1}}\right)\left(\int\left[\Gamma^{2}\right] \wedge \pi^{*}(\omega)^{k_{2}+l_{2}-s_{2}} \wedge \pi_{2}^{*}\left(\omega_{2}\right)^{s_{2}}\right)
$$

qui est égal à $\lambda_{s_{1}}\left(F_{1}\right) \lambda_{s_{2}}\left(F_{2}\right)$. La proposition en découle.

Soient $G_{i}: X_{i} \rightarrow X, i=1,2$, deux TM. On définit l'intersection $G_{1} \cap G_{2}$ de $G_{1}$ et $G_{2}$ comme l'adjoint $\bar{G}_{1} \bullet \bar{G}_{2}$ du produit $\bar{G}_{1} \bullet \bar{G}_{2}$ lorsque ce produit est bien défini. C'est une TM de $X_{1} \times X_{2}$ dans $X$. Pour $\left(x_{1}, x_{2}\right)$ générique, $\left(G_{1} \cap G_{2}\right)\left(x_{1}, x_{2}\right)$ est l'intersection de $G_{1}\left(x_{1}\right)$ et $G_{2}\left(x_{2}\right)$.

3.4. Familles de sous-ensembles analytiques. Soit $F^{\prime}$ une TM de codimension $l^{\prime}$ de $X_{2}$ dans $X_{3}$ dont le graphe est irréductible. La réunion de ses fibres $\mathscr{H}_{x_{3}}:=F^{\prime-1}\left(x_{3}\right)$ est égale à $X_{2}$. On dira que $\mathscr{H}=\left(\mathscr{H}_{x_{3}}\right)$ est une famille (méromorphe) adaptée d'ensembles analytiques de dimension $l^{\prime}$. Si $x_{3}$ n'appartient pas à $I_{2}\left(F^{\prime}\right), \mathscr{H}_{x_{3}}$ est de dimension $l^{\prime}$. Pour $x_{3}$ générique, les composantes de $\mathscr{H}_{x_{3}}$ sont de multiplicité 1 et on a $\left[\mathcal{H}_{x_{3}}\right]=\left(F^{\prime}\right)^{*}\left(\delta_{x_{3}}\right)$.

Soit $F$ une TM de codimension $l$ de $X_{1}$ dans $X_{2}$. Supposons que $l+l^{\prime}<k_{1}$. On dira que $\mathscr{H}$ est $F$-régulière si $\operatorname{dim} F^{-1}\left(\mathscr{H}_{x_{3}} \cap I_{2}(F)\right)<l+l^{\prime}$ pour $x_{3} \in X_{3}$ générique. Pour un tel $x_{3}, F^{*} F^{\prime *}\left(\delta_{x_{3}}\right)$ est bien défini. C'est un courant d'intégration sur une chaîne holomorphe de dimension $l+l^{\prime}$. La famille $\mathscr{H}$ est dite régulière, si elle est $F$-régulière pour toute TM $F$ d'une variété $X_{1}$ dans $X_{2}$. Les familles de sous-variétés associées aux TM $\Psi_{1}, \Psi_{2}, \Psi_{3}$ et $F_{l, n}$ que nous allons décrire au paragraphe 3.6 sont régulières. Les intersections de familles adaptées de sous-ensembles analytiques sont définies comme étant associées aux produits de TM.

3.5. Correspondances. Supposons que $X_{1}$ et $X_{2}$ ont la même dimension $k$. Une correspondance (méromorphe) de $X_{1}$ dans $X_{2}$ est une TM $f$ de codimension 0 de 
$X_{1}$ dans $X_{2}$. Notons $\Gamma=\sum \Gamma_{i}$ le graphe de $f$. La correspondance $\bar{f}$ de $X_{2}$ dans $X_{1}$ associée à $\bar{\Gamma}$ est appelée correspondance adjointe de $f$. Lorsque la restriction de $\pi_{1}$ à $\Gamma$ est injective hors d'un sous-ensemble analytique, on dira que $f$ est une application méromorphe dominante. On dit que $f$ est biméromorphe si $f$ et son adjoint $\bar{f}$ sont des applications méromorphes dominantes. Posons

$$
\mathrm{A}(f):=\sup _{\varphi}\left\{\left|\int_{X_{2}} f_{*}(\varphi) \omega_{2}^{k}\right|, \varphi \in \mathrm{Q}\left(X_{1}, \omega_{1}\right), \int \varphi \omega_{1}^{k}=0\right\} .
$$

D'après les résultats du paragraphe $2, \mathrm{~A}(f)$ est finie. Il mesure combien $f_{*}$ perturbe la normalisation $\int \varphi \omega_{1}^{k}=0$.

Considérons le cas où $X_{1}=X_{2}=X$. On notera $f^{n}$ la correspondance $f \circ \cdots \circ f$ ( $n$ fois). Pour tout $0 \leq s \leq k$, on définit le degré dynamique d'ordre $s$ de $f$ par la formule suivante :

$$
d_{s}(f):=\lim _{n \rightarrow \infty}\left[\lambda_{s}\left(f^{n}\right)\right]^{1 / n} .
$$

D'après la proposition 3.1, la suite $\left[\lambda_{s}\left(f^{n}\right)\right]^{1 / n}$ converge vers sa borne inférieure $\inf _{n \geq 1}\left[\lambda_{s}\left(f^{n}\right)\right]^{1 / n}$. Le dernier degré dynamique $d_{t}(f):=d_{k}(f)$ est égal au nombre d'éléments de la fibre $f^{-1}(z)$ pour $z$ générique (ce nombre ne dépend pas de $z$ ). C'est le degré topologique de $f$. On a aussi $d_{0}(f)=d_{t}(\bar{f})$.

3.6. Exemples. (a) Notons $\mathrm{G}(k-l+1, k+1)$ la grassmannienne qui paramètre les sous-espaces projectifs de dimension $k-l$ de $\mathbb{P}^{k}$. Pour $\hat{\boldsymbol{s}} \in \mathrm{G}(k-l+1, k+1)$, soit $\mathbb{P}_{\hat{\boldsymbol{s}}}^{k-l}$ le sous-espace projectif de dimension $k-l$ correspondant. Posons

$$
\Gamma_{1}:=\left\{(z, \hat{\boldsymbol{s}}) \in \mathbb{P}^{k} \times \mathrm{G}(k-l+1, k+1), z \in \mathbb{P}_{\hat{\boldsymbol{s}}}^{k-l}\right\} .
$$

La TM $\Psi_{1}$ de $\mathbb{P}^{k}$ dans $\mathrm{G}(k-l+1, k+1)$ associée à la variété $\Gamma_{1}$ est de codimension $k-l$ et on a $\Psi_{1}^{-1}(\hat{\boldsymbol{s}})=\mathbb{P}_{\hat{\boldsymbol{s}}}^{k-l}$.

Donnons une autre manière de voir cette TM. Soit $\mathbb{P}^{k *}:=\mathrm{G}(k, k+1)$ le dual de $\mathbb{P}^{k}$ et soit $\mathrm{G}^{*}(l, k+1)$ la grassmannienne qui paramètre les sous-espaces projectifs de dimension $l-1$ de $\mathbb{P}^{k *}$. Elle est biholomorphe à $\mathrm{G}(k-l+1, k+1)$. Pour tout $\check{\boldsymbol{s}} \in \mathrm{G}^{*}(l, k+1)$ notons $\mathbb{P}_{\check{\boldsymbol{s}}}^{(l-1) *}$ le sous-espace projectif de $\mathbb{P}^{k *}$ associé à $\check{s}$. On choisit $l$ points $s_{1}, \ldots, s_{l}$ de $\mathbb{P}_{\breve{s}}^{(l-1) *}$ qui engendrent $\mathbb{P}_{\breve{s}}^{(l-1) *}$. Notons $\mathbb{P}_{s_{i}}^{k-1} l^{\prime}$ 'hyperplan de $\mathbb{P}^{k}$ associé à $s_{i}$ et $\mathbb{P}_{\breve{s}}^{k-l}:=\mathbb{P}_{s_{1}}^{k-1} \cap \cdots \cap \mathbb{P}_{s_{l}}^{k-1}$. Le sous-espace projectif $\mathbb{P}_{\breve{s}}^{k-l}$ de $\mathbb{P}^{k}$ est indépendant du choix des $s_{i}$. Posons

$$
\Gamma_{2}:=\left\{(z, \check{\boldsymbol{s}}) \in \mathbb{P}^{k} \times \mathrm{G}^{*}(l, k+1), z \in \mathbb{P}_{\breve{\boldsymbol{s}}}^{k-l}\right\} .
$$

La TM $\Psi_{2}$ de $\mathbb{P}^{k}$ dans $\mathrm{G}^{*}(l, k+1)$ associée à $\Gamma_{2}$ est de codimension $k-l$ et on a $\Psi_{2}^{-1}(\check{\boldsymbol{s}})=\mathbb{P}_{\breve{\boldsymbol{s}}}^{k-l}$. 
Vol. 81 (2006) Distribution des valeurs de transformations méromorphes et applications 237

(b) Considérons l'espace multiprojectif $\mathbb{P}^{k, l *}:=\mathbb{P}^{k *} \times \cdots \times \mathbb{P}^{k *}$ (l fois). Posons

$\Gamma_{3}:=\left\{(\boldsymbol{s}, \check{\boldsymbol{s}}) \in \mathbb{P}^{k, l *} \times \mathrm{G}^{*}(l, k+1), \boldsymbol{s}=\left(s_{1}, \ldots, s_{l}\right), \mathbb{P}_{\breve{s}}^{k-l} \subset \mathbb{P}_{s_{i}}^{k-1}\right.$ pour $\left.i=1, \ldots, l\right\}$.

Notons $\Pi_{l}$ la TM de $\mathbb{P}^{k, l *}$ dans $\mathrm{G}^{*}(l, k+1)$ associée à $\Gamma_{3}$. C'est une application méromorphe dominante. Soit $\bar{\Pi}_{l}$ son adjoint. La composée $\Psi_{3}:=\bar{\Pi}_{l} \circ \Psi_{2}$ est une TM de $\mathbb{P}^{k}$ dans $\mathbb{P}^{k, l *}$. Pour $\boldsymbol{s}=\left(s_{1}, \ldots, s_{l}\right)$ générique dans $\mathbb{P}^{k, l *}$, la fibre $\Psi_{3}^{-1}(\boldsymbol{s})$ est le sous-espace projectif $\mathbb{P}_{s}^{k-l}:=\mathbb{P}_{s_{1}}^{k-1} \cap \cdots \cap \mathbb{P}_{s_{l}}^{k-1}$ de $\mathbb{P}^{k}$.

(c) Nous allons étendre la définition des TM de (a) et (b) avec, pour espace d'arrivée, un espace projectif de sections holomorphes. La construction permettra de calculer facilement les degrés intermédiaires (voir lemme 7.1). Considérons une variété projective $X$ et soit $L$ un fibré en droites ample sur $X$. Notons $\mathrm{H}^{0}\left(X, L^{n}\right)$ l'espace des sections holomorphes de $L^{n}:=L \otimes \cdots \otimes L$ ( $n$ fois), $\mathbb{P H}^{0}\left(X, L^{n}\right)$ l'espace projectif associé et $k_{n}$ sa dimension. Pour tout $s^{*} \in \mathbb{P H}^{0}\left(X, L^{n}\right)^{*}$ notons $H_{s^{*}}$ l'hyperplan projectif de $\mathbb{P H}^{0}\left(X, L^{n}\right)$ associé à $s^{*}$. Rappelons que $\mathbb{P H}^{0}\left(X, L^{n}\right)$ est aussi le dual de $\mathbb{P} H^{0}\left(X, L^{n}\right)^{*}$. Pour tout $s \in \mathbb{P H}^{0}\left(X, L^{n}\right)$, notons $H_{s}^{*} l^{\prime}$ hyperplan de $\mathbb{P} \mathrm{H}^{0}\left(X, L^{n}\right)^{*}$ associé à $s$.

Pour $x \in X$, notons $s_{x}^{*} \in \mathbb{P H}^{0}\left(X, L^{n}\right)^{*}$ le point tel que l'hyperplan $H_{S_{x}^{*}}$ soit l'ensemble des sections s'annulant en $x$. Considérons l'application holomorphe $\Phi_{n}$ de $X$ dans $\mathbb{P} H^{0}\left(X, L^{n}\right)^{*}$ définie par $x \mapsto \Phi_{n}(x):=s_{x}^{*}$. Puisque $L$ est ample, pour $n$ assez grand, $\Phi_{n}$ définit un plongement de $X$ dans $\mathbb{P H}^{0}\left(X, L^{n}\right)^{*}$, c'est le plongement de Kodaira. Observons que $\Phi_{n}^{-1}\left(H_{s}^{*} \cap \Phi_{n}(X)\right)$ est l'ensemble des zéros de $s$. C'est une hypersurface lisse de $X$ et l'intersection $H_{s}^{*} \cap \Phi_{n}(X)$ est transverse pour tout $s$ hors d'un sous-ensemble analytique de $\mathbb{P H}^{0}\left(X, L^{n}\right)$.

Soit $\mathrm{G}_{l, n}^{X}$ la grassmannienne des sous-espaces projectifs de dimension $l-1$ de $\mathbb{P H}^{0}\left(X, L^{n}\right)$. On construit comme dans (a) une $\mathrm{TM} \Psi_{l, n}$ de $\mathbb{P} \mathrm{H}^{0}\left(X, L^{n}\right)^{*}$ dans $\mathrm{G}_{l, n}^{X}$. Pour tout point $\check{\boldsymbol{s}} \in \mathrm{G}_{l, n}^{X}, \Psi_{l, n}^{-1}(\check{\boldsymbol{s}})$ est un sous-espace projectif de dimension $k_{n}-l \mathrm{de}$ $\mathbb{P H}^{0}\left(X, L^{n}\right)^{*}$. Posons $R_{l, n}:=\Psi_{l, n} \circ \Phi_{n}$. C'est une TM de codimension $k-l$ de $X$ dans $\mathrm{G}_{l, n}^{X}$. Précisons cela.

Notons $s_{1}, \ldots, s_{l}$ des points qui engendrent le sous-espace $\mathbb{P}_{\breve{s}}^{l-1}$ de dimension $l-1$ de $\mathbb{P} H^{0}\left(X, L^{n}\right)$, pour $\check{\boldsymbol{s}} \in \mathrm{G}_{l, n}^{X}$. Alors $\Psi_{l, n}^{-1}(\check{\boldsymbol{s}})$ est égal au sous-espace projectif $H_{s_{1}}^{*} \cap \cdots \cap H_{s_{l}}^{*}$ de dimension $k_{n}-l \operatorname{de} \mathbb{P} H^{0}\left(X, L^{n}\right)^{*}$. Donc $R_{l, n}^{-1}(\check{\boldsymbol{s}})$ est l'ensemble $\mathbf{Z}_{\check{s}}$ des zéros communs des sections $s_{1}, \ldots, s_{l}$. Il ne dépend pas du choix des $s_{i}$. Pour $\check{\boldsymbol{s}} \in \mathrm{G}_{l, n}^{X}$ hors d'un sous-ensemble analytique, l'intersection $\Psi_{l, n}^{-1}(\check{\boldsymbol{s}}) \cap \Phi_{n}(X)$ est transverse et $\mathbf{Z}_{\check{s}}$ est lisse.

Soit $\Pi_{l, n}$ l'application méromorphe de $\mathbb{P}_{l, n}^{X}:=\mathbb{P H}^{0}\left(X, L^{n}\right) \times \cdots \times \mathbb{P H}^{0}\left(X, L^{n}\right)$ ( $l$ fois) dans $\mathrm{G}_{l, n}^{X}$ définie comme dans (b). Soit $\bar{\Pi}_{l, n}$ sont adjoint. Posons $F_{l, n}:=$ $\bar{\Pi}_{l, n} \circ R_{l, n}$. C'est une TM de $X$ dans $\mathbb{P}_{l, n}^{X}$. Pour tout $s=\left(s_{1}, \ldots, s_{l}\right) \in \mathbb{P}_{l, n}^{X}$, la fibre $F_{l, n}^{-1}(s)$ est l'ensemble des zéros communs de $s_{1}, \ldots, s_{l}$. Pour un $s$ générique, 
cette fibre est égale à $\mathbf{Z}_{\check{s}}$ avec $\check{\boldsymbol{s}}:=\Pi_{l, n}(\boldsymbol{s})$. En particulier, c'est un sous-ensemble analytique lisse de dimension $k-l$, sans multiplicité.

(d) Soit $f$ une application méromorphe de $\mathbb{P}^{k}$ dans $\mathbb{P}^{k}$. Pour étudier les images réciproques par $f^{n}$ des sous-espaces de dimension $k-l$, nous introduisons les TM $F_{n}:=\Psi_{2} \circ f^{n}$ avec $\Psi_{2}$ définie dans (a). On se ramène à l'étude des images réciproques des points de $\mathrm{G}^{*}(l, k+1)$ par $F_{n}$.

\section{Distribution des préimages de sous-variétés}

Soit $\sigma_{n}$ une mesure de probabilité PB sur $X_{n}$. On munit $X:=\prod_{n \geq 1} X_{n}$ de la mesure de probabilité $\sigma$, égale au produit des $\sigma_{n}$. Considérons une suite de TM $F_{n}: X \rightarrow X_{n}$ de même codimension $l, 0 \leq l<k$. Soit $\boldsymbol{x}=\left(x_{1}, x_{2}, \ldots\right) \in \boldsymbol{X}$. Si $x_{n}$ n'appartient pas à $I_{2}\left(F_{n}\right), T_{n}^{x}:=\left(F_{n}\right)^{*}\left(\delta_{x_{n}}\right)$ est bien défini. C'est un courant d'intégration sur une chaîne holomorphe de dimension $l$ de $X$. Posons $T_{n}:=\left(F_{n}\right)^{*}\left(\sigma_{n}\right), \mathrm{R}_{i, n}:=\mathrm{R}_{i}\left(X_{n}, \omega_{n}, \sigma_{n}\right)$ et $\Delta_{n}(t):=\Delta\left(X_{n}, \omega_{n}, \sigma_{n}, t\right)$. Soient $\delta_{n}$ et $d_{n}$ les degrés intermédiaires d'ordre $k_{n}-1$ et d'ordre $k_{n}$ de $F_{n}$.

Théorème 4.1. Supposons que la suite $\left(\mathrm{R}_{1, n} \delta_{n} d_{n}^{-1}\right)_{n \geq 1}$ tend vers 0 et que l'une des deux propriétés suivantes soit satisfaite :

(1) La série $\sum_{n \geq 1} \mathrm{R}_{2, n} \delta_{n} d_{n}^{-1}$ converge.

(2) La série $\sum_{n \geq 1} \Delta_{n}\left(\delta_{n}^{-1} d_{n} t\right)$ converge pour tout $t>0$.

Alors pour $\sigma$-presque tout $\boldsymbol{x} \in \boldsymbol{X}$, la suite $\left\langle d_{n}^{-1}\left(T_{n}^{\boldsymbol{x}}-T_{n}\right), \psi\right\rangle$ tend vers 0 uniformément sur les ensembles bornés, en norme $\mathcal{C}^{2}$, de $(l, l)$-formes test $\psi$ sur $X$.

Nous allons montrer les estimations utiles en nous limitant à une TM $F: X \rightarrow$ $X^{\prime}$ de codimension $l, 0 \leq l \leq k-1$. Soient $\sigma^{\prime}$ une mesure de probabilité PB sur $X^{\prime}, \delta$ et $d$ les degrés intermédiaires d'ordre $k^{\prime}-1$ et d'ordre $k^{\prime}$ de $F$. Posons $\widetilde{T}:=F^{*}\left(\omega^{\prime k^{\prime}}\right), T:=F^{*}\left(\sigma^{\prime}\right)$ et $T^{x^{\prime}}:=F^{*}\left(\delta_{x^{\prime}}\right)$ pour $x^{\prime} \in X^{\prime} \backslash I_{2}(F)$. Posons aussi $\mathrm{R}_{i}:=\mathrm{R}_{i}\left(X^{\prime}, \omega^{\prime}, \sigma^{\prime}\right), \Delta(t):=\Delta\left(X^{\prime}, \omega^{\prime}, \sigma^{\prime}, t\right)$ et pour tout $\varepsilon>0$

$$
E(\varepsilon):=\bigcup_{\|\psi\|_{\mathcal{C}^{2}} \leq 1}\left\{x^{\prime} \in X^{\prime},\left|\left\langle d^{-1}\left(T^{x^{\prime}}-T\right), \psi\right\rangle\right| \geq \varepsilon\right\} .
$$

Soit $S:=F_{*}\left(\omega^{l+1}\right)$. C'est un courant de bidegré $(1,1)$ et de masse $\delta$ sur $X^{\prime}$. D'après la proposition 2.2, il existe une fonction quasi-psh $\varphi$ vérifiant

$$
\int_{X^{\prime}} \varphi \mathrm{d} \sigma^{\prime}=0 \quad \text { et } \quad \mathrm{dd}^{\mathrm{c}} \varphi-S \geq-r\left(X^{\prime}, \omega^{\prime}\right) \delta \omega^{\prime}
$$


Vol. 81 (2006) Distribution des valeurs de transformations méromorphes et applications 239

Par définition de $\mathrm{R}_{i}$, on a

$$
\varphi \leq \delta \mathrm{R}_{1}, \quad\|\varphi\|_{\mathrm{L}^{1}\left(\sigma^{\prime}\right)} \leq \delta \mathrm{R}_{2} \quad \text { et } \quad\left|\int \varphi \omega^{\prime k^{\prime}}\right| \leq \delta \mathrm{R}_{3} .
$$

Lemme 4.2. Soit $\psi$ une $(l, l)$-forme test de classe $\mathcal{C}^{2}$ sur $X$ avec $\|\psi\|_{\mathfrak{C}^{2}}=1$. Alors on $a$
(a) $\int_{X^{\prime}}\left|\left\langle T^{x^{\prime}}-T, \psi\right\rangle\right| \mathrm{d} \sigma^{\prime}\left(x^{\prime}\right) \leq 2 \delta \mathrm{R}_{2}$.
(b) $\left|\left\langle T^{x^{\prime}}-T, \psi\right\rangle\right| \leq 3 \delta \mathrm{R}_{1}-\varphi\left(x^{\prime}\right)$.
(c) $|\langle T-\widetilde{T}, \psi\rangle| \leq 2 \delta \mathrm{R}_{3}$.
(d) $\sigma^{\prime}(E(\varepsilon)) \leq \Delta\left(\varepsilon \delta^{-1} d-3 \mathrm{R}_{1}\right)$.

Démonstration. Nous devons estimer $\left\langle T^{x^{\prime}}-T, \psi\right\rangle$ et $\langle T-\widetilde{T}, \psi\rangle$. Ecrivons $\mathrm{dd}^{\mathrm{c}} \psi=$ $\Omega^{+}-\Omega^{-}$avec des $(l+1, l+1)$-formes $\Omega^{ \pm}$positives fermées telles que $\Omega^{ \pm} \leq \omega^{l+1}$. Posons $\phi:=F_{*}(\psi)$ et $S^{ \pm}:=F_{*}\left(\Omega^{ \pm}\right)$. On a dd $\mathrm{dd}^{\mathrm{c}} \phi=S^{+}-S^{-}$et $\left\langle T^{x^{\prime}}, \psi\right\rangle=\phi\left(x^{\prime}\right)$ pour $x^{\prime} \notin I_{2}(F)$. On a aussi $S^{ \pm} \leq S$ et donc $\left\|S^{ \pm}\right\| \leq \delta$. D'après la proposition 2.2, il existe des fonctions quasi-psh $\varphi^{ \pm}$vérifiant $\int_{X^{\prime}} \varphi^{ \pm} \mathrm{d} \sigma^{\prime}=0$ telles que

$$
-r\left(X^{\prime}, \omega^{\prime}\right) \delta \omega^{\prime} \leq \operatorname{dd}^{\mathrm{c}} \varphi^{+}-S^{+}=\mathrm{dd}^{\mathrm{c}} \varphi^{-}-S^{-} \leq r\left(X^{\prime}, \omega^{\prime}\right) \delta \omega^{\prime} .
$$

(pour être exact, il faut remplacer $r\left(X^{\prime}, \omega^{\prime}\right)$ par $r\left(X^{\prime}, \omega^{\prime}\right)+\varepsilon^{\prime}, \varepsilon^{\prime}>0$, mais notre abus ne change pas les résultats car on peut ensuite faire tendre $\varepsilon^{\prime}$ vers 0$)$. Par définition des $\mathrm{R}_{i}$ on a

$$
\varphi^{ \pm} \leq \delta \mathbf{R}_{1}, \quad\left\|\varphi^{ \pm}\right\|_{L^{1}\left(\sigma^{\prime}\right)} \leq \delta \mathbf{R}_{2} \quad \text { et } \quad\left|\int \varphi^{ \pm} \omega^{\prime k^{\prime}}\right| \leq \delta \mathbf{R}_{3} .
$$

La fonction $\phi-\left(\varphi^{+}-\varphi^{-}\right)$est constante car elle est pluriharmonique. De plus, comme $\int \varphi^{ \pm} \mathrm{d} \sigma^{\prime}=0$, on obtient pour $x^{\prime} \notin I_{2}(F)$

$$
\left\langle T^{x^{\prime}}-T, \psi\right\rangle=\left\langle\delta_{x^{\prime}}-\sigma^{\prime}, \phi\right\rangle=\left\langle\delta_{x^{\prime}}-\sigma^{\prime}, \varphi^{+}-\varphi^{-}\right\rangle=\varphi^{+}\left(x^{\prime}\right)-\varphi^{-}\left(x^{\prime}\right) .
$$

(a) On en déduit que

$$
\int_{X^{\prime}}\left|\left\langle T^{x^{\prime}}-T, \psi\right\rangle\right| \mathrm{d} \sigma^{\prime}\left(x^{\prime}\right) \leq\left\|\varphi^{+}\right\|_{\mathrm{L}^{1}\left(\sigma^{\prime}\right)}+\left\|\varphi^{-}\right\|_{\mathrm{L}^{1}\left(\sigma^{\prime}\right)} \leq 2 \delta \mathrm{R}_{2} .
$$

(b) Posons $h:=\varphi-\varphi^{+}$. Comme $S^{ \pm} \leq S$, d'après (4.1) et (4.2), on a

$$
\mathrm{dd}^{\mathrm{c}} h \geq-2 r\left(X^{\prime}, \omega^{\prime}\right) \delta \omega^{\prime} .
$$

D'autre part, on a $\int_{X^{\prime}} h \mathrm{~d} \sigma^{\prime}=0$. Par définition de $\mathrm{R}_{1}, \max _{X^{\prime}} h \leq 2 \delta \mathrm{R}_{1}$. Ceci et (4.3) entraînent que

$$
\varphi\left(x^{\prime}\right)-2 \delta \mathrm{R}_{1} \leq \varphi^{+}\left(x^{\prime}\right) \leq \delta \mathrm{R}_{1} .
$$


Avec une estimation analogue pour $\varphi^{-}$, on obtient

$$
\left|\left\langle T^{x^{\prime}}-T, \psi\right\rangle\right|=\left|\varphi^{+}\left(x^{\prime}\right)-\varphi^{-}\left(x^{\prime}\right)\right| \leq 3 \delta \mathrm{R}_{1}-\varphi\left(x^{\prime}\right) .
$$

(c) D'après (4.3), on a

$$
\begin{aligned}
|\langle T-\widetilde{T}, \psi\rangle| & =\left|\left\langle\sigma-\omega^{\prime k^{\prime}}, \varphi^{+}-\varphi^{-}\right\rangle\right|=\left|\left\langle\omega^{\prime k^{\prime}}, \varphi^{+}-\varphi^{-}\right\rangle\right| \\
& \leq\left|\left\langle\omega^{\prime k^{\prime}}, \varphi^{+}\right\rangle\right|+\left|\left\langle\omega^{\prime k^{\prime}}, \varphi^{-}\right\rangle\right| \leq 2 \delta \mathrm{R}_{3} .
\end{aligned}
$$

(d) D'après (b), $E(\varepsilon)$ est contenu dans

$$
E^{\prime}(\varepsilon):=\left\{x^{\prime} \in X^{\prime}, \varphi\left(x^{\prime}\right) \leq-\varepsilon d+3 \delta \mathrm{R}_{1}\right\} .
$$

Par définition de $\Delta(t)$, les relations (4.1) entrainent que

$$
\sigma^{\prime}(E(\varepsilon)) \leq \sigma^{\prime}\left(E^{\prime}(\varepsilon)\right) \leq \Delta\left(\varepsilon \delta^{-1} d-3 \mathrm{R}_{1}\right) .
$$

Démonstration du théorème 4.1. Posons $S_{n}:=\left(F_{n}\right)_{*}\left(\omega^{l+1}\right)$. On a $\left\|S_{n}\right\|=\delta_{n}$. D'après la proposition 2.2, il existe $\varphi_{n}$ quasi-psh vérifiant

$$
\int_{X_{n}} \varphi_{n} \mathrm{~d} \sigma_{n}=0 \quad \text { et } \quad \mathrm{dd}^{\mathrm{c}} \varphi_{n}-S_{n} \geq-r\left(X_{n}, \omega_{n}\right) \delta_{n} \omega_{n} .
$$

Par définition de $\mathrm{R}_{i, n}$, on a $\varphi_{n} \leq \delta_{n} \mathrm{R}_{1, n}$ et $\left\|\varphi_{n}\right\|_{\mathrm{L}^{1}\left(\sigma_{n}\right)} \leq \delta_{n} \mathrm{R}_{2, n}$.

(1) Considérons la fonction réelle positive $\Phi$ sur $\boldsymbol{X}$

$$
\Phi(\boldsymbol{x}):=\sum_{n \geq 1} d_{n}^{-1}\left|\varphi_{n}\left(x_{n}\right)\right|
$$

On a

$$
\int_{X} \Phi \mathrm{d} \sigma=\sum_{n \geq 1} d_{n}^{-1}\left\|\varphi_{n}\right\|_{\mathrm{L}^{1}\left(\sigma_{n}\right)} \leq \sum_{n \geq 1} \mathrm{R}_{2, n} \delta_{n} d_{n}^{-1} .
$$

Par hypothèse, la dernière série converge, donc $\Phi(\boldsymbol{x})$ est finie et $d_{n}^{-1} \varphi_{n}\left(x_{n}\right)$ tend vers 0 pour $\sigma$-presque tout $\boldsymbol{x} \in \boldsymbol{X}$. Fixons $\boldsymbol{x}=\left(x_{n}\right) \in \boldsymbol{X}$ tel que $x_{n} \notin I_{2}\left(F_{n}\right)$ et tel que $d_{n}^{-1} \varphi_{n}\left(x_{n}\right)$ tende vers 0 . Soit $\psi$ une $(l, l)$-forme de classe $\mathcal{C}^{2}$ sur $X$. D'après le lemme 4.2(b), on a

$$
\left|\left\langle T_{n}^{x}-T_{n}, \psi\right\rangle\right| \leq\|\psi\|_{\mathcal{C}^{2}}\left(3 \delta_{n} \mathrm{R}_{1, n}-\varphi_{n}\left(x_{n}\right)\right) .
$$

On déduit de l'hypothèse la convergence souhaitée.

(2) Posons pour tout $\varepsilon>0$

$$
E_{n}(\varepsilon):=\bigcup_{\|\psi\|_{\mathcal{C}^{2}} \leq 1}\left\{x_{n} \in X_{n},\left|\left\langle d_{n}^{-1}\left(T_{n}^{x}-T_{n}\right), \psi\right\rangle\right| \geq \varepsilon\right\} .
$$


Vol. 81 (2006) Distribution des valeurs de transformations méromorphes et applications 241

Par hypothèse, $\mathrm{R}_{1, n}=\mathrm{o}\left(\delta_{n}^{-1} d_{n}\right)$. D'après le lemme 4.2(d), on obtient pour $n$ assez grand que

$$
\sigma_{n}\left(E_{n}(\varepsilon)\right) \leq \Delta_{n}\left(\varepsilon \delta_{n}^{-1} d_{n}-3 \mathrm{R}_{1, n}\right) \leq \Delta_{n}\left(\varepsilon \delta_{n}^{-1} d_{n} / 2\right) .
$$

La série $\sum \Delta\left(\varepsilon \delta_{n}^{-1} d_{n} / 2\right)$ étant convergeante, $\sum \sigma_{n}\left(E_{n}(\varepsilon)\right)$ converge aussi pour tout $\varepsilon>0$. Ceci implique la convergence annoncée.

La proposition suivante permet de comparer les courants obtenus en prenant les images réciproques de $\sigma_{n}$ et de la forme volume $\omega_{n}^{k_{n}}$.

Proposition 4.3. Supposons que la suite $\mathrm{R}_{3, n} \delta_{n} d_{n}^{-1}$ tend vers 0 . Alors $\left\langle d_{n}^{-1}\left(T_{n}-\right.\right.$ $\left.\left.F_{n}^{*}\left(\omega_{n}^{k_{n}}\right)\right), \psi\right\rangle$ tend vers 0 uniformément sur les ensembles bornés, en norme $\mathcal{C}^{2}$, de $(l, l)$-formes $\psi$ sur $X$.

Démonstration. Il suffit d'appliquer le lemme 4.2(c) avec les estimations comme dans la démonstration du théorème 4.1.

Posons $\mathrm{R}_{n}^{*}:=\mathrm{R}_{2}^{*}\left(X_{n}, \omega_{n}, 2\right)$. Soient $v_{n}=h_{n} \omega_{n}^{k_{n}}$ et $v_{n}^{\prime}=h_{n}^{\prime} \omega_{n}^{k_{n}}$ des mesures de probabilité sur $X_{n}$ où $h_{n}$ et $h_{n}^{\prime}$ sont des fonctions dans $\mathrm{L}^{2}\left(X_{n}\right)$.

Théorème 4.4. Supposons que $\left\|h_{n}-h_{n}^{\prime}\right\|_{\mathrm{L}^{2}\left(X_{n}\right)}=\mathrm{o}\left(\delta_{n}^{-1} d_{n} \mathrm{R}_{n}^{*-1}\right)$. Alors

$$
d_{n}^{-1}\left\langle\left(F_{n}\right)^{*}\left(v_{n}\right)-\left(F_{n}\right)^{*}\left(v_{n}^{\prime}\right), \psi\right\rangle
$$

tend vers 0 uniformément sur les ensembles bornés, en norme $\mathcal{C}^{2}$, de $(l, l)$-formes test $\psi$ sur $X$.

Démonstration. Utilisons les notations du théorème 4.1. Posons $\phi_{n}:=\left(F_{n}\right)_{*}(\psi)$. Il existe des constantes $a_{n}$ et des fonctions quasi-psh $\varphi_{n}^{ \pm}$telles que $\phi_{n}=\varphi_{n}^{+}-\varphi_{n}^{-}+a_{n}$ et $\left\|\varphi_{n}^{ \pm}\right\|_{\mathrm{L}^{2}\left(X_{n}\right)} \leq\|\psi\|_{\mathcal{C}^{2}} \mathrm{R}_{n}^{*} \delta_{n}$. En utilisant l'inégalité de Cauchy-Schwarz et le fait que $v_{n}$ et $v_{n}^{\prime}$ ont la même masse, on obtient

$$
\begin{aligned}
& d_{n}^{-1}\left|\left\langle\left(F_{n}\right)^{*}\left(v_{n}\right)-\left(F_{n}\right)^{*}\left(v_{n}^{\prime}\right), \psi\right\rangle\right|=d_{n}^{-1}\left|\left\langle\left(h_{n}-h_{n}^{\prime}\right) \omega_{n}^{k_{n}}, \phi_{n}-a_{n}\right\rangle\right| \\
& \leq d_{n}^{-1}\left\|h_{n}-h_{n}^{\prime}\right\|_{\mathrm{L}^{2}\left(X_{n}\right)}\left\|\phi_{n}-a_{n}\right\|_{\mathrm{L}^{2}\left(X_{n}\right)} \\
& \leq 2\|\psi\|_{\mathcal{C}^{2}} \delta_{n} d_{n}^{-1} \mathrm{R}_{n}^{*}\left\|h_{n}-h_{n}^{\prime}\right\|_{\mathrm{L}^{2}\left(X_{n}\right)} \text {. }
\end{aligned}
$$

Par hypothèse, la dernière expression tend vers 0 .

Remarque 4.5. Si les $\left(X_{n}, \omega_{n}, \sigma_{n}\right)$ appartiennent à une famille lisse $\left(X_{t}\right)$ de variétés kählériennes compactes, d'après Kodaira-Spencer [16, p. 73], $\operatorname{dim} H^{1,1}\left(X_{t}, \mathbb{C}\right)$ est localement constante et les $r\left(X_{t}, \omega_{t}\right)$ sont localement majorées. Il en résulte que les $\mathrm{R}_{1, n}$ et $\mathrm{R}_{n}^{*}$ sont uniformément bornées. Les hypothèses des théorèmes 4.1 et 4.4 ne font alors intervenir que les degrés intermédiaires de $F_{n}$ qui sont calculés cohomologiquement. 
Dans la suite, nous considérons des TM $F_{n}$ de $X$ dans une même variété $X^{\prime}$, c.-à-d. qu'on suppose que $\left(X_{n}, \omega_{n}\right)=\left(X^{\prime}, \omega^{\prime}\right)$ pour tout $n \geq 1$.

Théorème 4.6. Soit $\sigma^{\prime}$ une mesure de probabilité $P B$ sur $X^{\prime}$. Supposons que $\sum_{n \geq 1} \Delta\left(X^{\prime}, \omega^{\prime}, \sigma^{\prime}, t \delta_{n}^{-1} d_{n}\right)$ converge pour tout $t>0$. Alors pour $\sigma^{\prime}$-presque tout $x^{\prime} \in X^{\prime},\left\langle d_{n}^{-1}\left(\left(F_{n}\right)^{*}\left(\delta_{x^{\prime}}\right)-\left(F_{n}\right)^{*}\left(\omega^{\prime k^{\prime}}\right)\right), \psi\right\rangle$ tend vers 0 uniformément sur les ensembles bornés, en norme $\mathcal{C}^{2}$, de $(l, l)$-formes test $\psi$ sur $X$.

Démonstration. Posons $r:=r\left(X^{\prime}, \omega^{\prime}\right)$. Pour tout $\varepsilon>0$, posons

$$
E_{n}(\varepsilon):=\bigcup_{\|\psi\|_{\mathcal{C}^{2}} \leq 1}\left\{x^{\prime} \in X^{\prime},\left|\left\langle d_{n}^{-1}\left(F_{n}^{*}\left(\delta_{x^{\prime}}\right)-F_{n}^{*}\left(\omega^{\prime k^{\prime}}\right)\right), \psi\right\rangle\right| \geq \varepsilon\right\},
$$

et $\mathrm{R}_{1}:=\mathrm{R}_{1}\left(X^{\prime}, \omega^{\prime}, \sigma^{\prime}\right), \Delta(t):=\Delta\left(X^{\prime}, \omega^{\prime}, \sigma^{\prime}, t\right)$. Le lemme 4.2(d) entraîne que $\sigma^{\prime}\left(E_{n}(\varepsilon)\right) \leq \Delta\left(\varepsilon \delta_{n}^{-1} d_{n}-3 \mathrm{R}_{1}\right)$. On déduit de l'hypothèse que $\lim \delta_{n}^{-1} d_{n}=+\infty$ et que la série $\sum_{n \geq 1} \Delta\left(\varepsilon \delta_{n}^{-1} d_{n}-3 \mathrm{R}_{1}\right)$ converge. Donc la série $\sum_{n \geq 1} \sigma^{\prime}\left(E_{n}(\varepsilon)\right)$ converge pour tout $\varepsilon>0$. Le théorème en découle.

Démonstration du théorème 1.1. (1) Posons $\mathrm{R}_{1}^{*}:=\mathrm{R}_{1}^{*}\left(X^{\prime}, \omega^{\prime}\right), \mathrm{R}_{2}^{*}:=\mathrm{R}_{2}^{*}\left(X^{\prime}, \omega^{\prime}, 1\right)$, $S_{n}:=\left(F_{n}\right)_{*}\left(\omega^{l+1}\right)$. On a $\left\|S_{n}\right\|=\delta_{n}$. D'après la proposition 2.2 , il existe $\varphi_{n}$ quasi-psh vérifiant

$$
\int_{X^{\prime}} \varphi_{n} \omega^{\prime k^{\prime}}=0 \quad \text { et } \quad \mathrm{dd}^{\mathrm{c}} \varphi_{n}-S_{n} \geq-r \delta_{n} \omega^{\prime} .
$$

Par définition de $\mathrm{R}_{i}^{*}$, on a $\varphi_{n} \leq \mathrm{R}_{1}^{*} \delta_{n}$ et $\left\|\varphi_{n}\right\|_{\mathrm{L}^{1}\left(X^{\prime}\right)} \leq \mathrm{R}_{2}^{*} \delta_{n}$. On en déduit que la série

$$
\Phi\left(x^{\prime}\right):=\sum_{n \geq 1} d_{n}^{-1} \varphi_{n}\left(x^{\prime}\right)
$$

converge ponctuellement vers une fonction quasi-psh. Posons

$$
\mathcal{E}:=\bigcup_{n \geq 1} I_{2}\left(F_{n}\right) \cup(\Phi=-\infty)
$$

D'après la proposition A. $1, \mathcal{E}$ est pluripolaire. Pour $x^{\prime} \in X^{\prime} \backslash \mathscr{E}$, on a $\lim d_{n}^{-1} \varphi_{n}\left(x^{\prime}\right)=0$. Le lemme 4.2(b) appliqué à la mesure $\sigma^{\prime}:=\omega^{\prime k^{\prime}}$, implique que $\left\langle d_{n}^{-1}\left(\left(F_{n}\right)^{*}\left(\delta_{x^{\prime}}\right)-\right.\right.$ $\left.\left.\left(F_{n}\right)^{*}\left(\omega^{\prime k^{\prime}}\right)\right), \psi\right\rangle$ tend vers 0 uniformément sur les ensembles bornés, en norme $\mathcal{C}^{2}$, de $(l, l)$-formes test $\psi$.

(2) La mesure $\sigma^{\prime}$ étant $(c, \alpha)$-modérée, la proposition 2.4 entraîne que

$$
\Delta\left(t \delta_{n}^{-1} d_{n}\right) \leq c \exp \left(-\alpha r^{-1} t \delta_{n}^{-1} d_{n}\right) .
$$

Par conséquent, la série $\sum \Delta\left(t \delta_{n}^{-1} d_{n}\right)$ converge pour tout $t>0$. Il suffit d'appliquer le théorème 4.6. 
Vol. 81 (2006) Distribution des valeurs de transformations méromorphes et applications 243

Soit $\mathscr{H}=\left(\mathscr{H}_{y}\right)_{y \in Y}$ une famille adaptée de sous-ensembles analytiques de dimension $m$ de $X^{\prime}$ associée à une TM $G: X^{\prime} \rightarrow Y$. Supposons que $l+m<k$ et que $\mathscr{H}$ soit $F_{n}$-régulière pour tout $n$. On a vu au paragraphe 2.4 que pour $y \in Y$ générique, les courants $\left[\lambda_{k^{\prime}-m}\left(F_{n}\right)\right]^{-1}\left(F_{n}\right)^{*}\left[\mathscr{H}_{y}\right]$ sont bien définis et de masse bornée indépendemment de $n$.

Corollaire 4.7. Supposons que la série $\sum_{n \geq 1} \lambda_{k^{\prime}-m-1}\left(F_{n}\right)\left[\lambda_{k^{\prime}-m}\left(F_{n}\right)\right]^{-1}$ converge. Alors la suite de courants

$$
\frac{1}{\lambda_{k^{\prime}-m}\left(F_{n}\right)}\left(\left(F_{n}\right)^{*}\left[\mathscr{H}_{y}\right]-\left(F_{n}\right)^{*}\left[\mathscr{H}_{y^{\prime}}\right]\right)
$$

tend faiblement vers 0 pour y et $y^{\prime}$ hors d'un ensemble pluripolaire $\& \subset Y$.

Démonstration. Il suffit d'appliquer le théorème 4.6 et la proposition 3.1 pour les TM $G \circ F_{n}$, puisque les $\mathscr{H}_{y}$ sont les fibres de $G$.

Remarque 4.8. Lorsque les $F_{n}$ sont des applications rationnelles entre les espaces projectifs et $\mathscr{H}$ est une famille de sous-espaces projectifs, ce résultat a été prouvé par Sodin, Russakovkii et Shiffman [21], [20].

\section{Mesures d'équilibre de correspondances}

Dans ce paragraphe, on suppose que les $X_{n}$ ont la même dimension $k$. Nous étudions l'itération aléatoire d'une suite de correspondances $f_{n}: X_{n-1} \rightarrow X_{n}$. Notons $d_{n}$ le degré topologique de $f_{n}, \delta_{n}$ le degré intermédiaire d'ordre $k-1$ de $f_{n} \circ \cdots \circ f_{1}, \mathrm{R}_{n}^{*}:=$ $\mathrm{R}_{2}^{*}\left(X_{n}, \omega_{n}, 2\right)$ et $\mathrm{A}_{n}:=\mathrm{A}\left(f_{n}\right)$. Soient $h_{n}$ des fonctions positives dans $\mathrm{L}^{2}\left(X_{n}\right)$ telles que $\int_{X_{n}} h_{n} \omega_{n}^{k}=1$. Posons $v_{n}:=h_{n} \omega_{n}^{k}$ et $\mu_{n}:=d_{1}^{-1} \ldots d_{n}^{-1}\left(f_{n} \circ \cdots \circ f_{1}\right)^{*}\left(v_{n}\right)$. Les mesures de probabilité $v_{n}$ et $\mu_{n}$ sont absolument continues par rapport aux mesures de Lebesgue.

Théorème 5.1. Supposons que $\delta_{n} \mathrm{R}_{n}^{*}\left\|h_{n}\right\|_{\mathrm{L}^{2}\left(X_{n}\right)}=\mathrm{o}\left(d_{1} \ldots d_{n}\right)$ et que de plus la série $\sum_{n \geq 2} d_{1}^{-1} \ldots d_{n}^{-1} \delta_{n-1} \mathrm{~A}_{n}$ converge. Alors $\mu_{n}$ tend faiblement vers une mesure de probabilité $P C \mu$ sur $X_{0}$. De plus, $\mu$ est indépendante de la suite $\left(h_{n}\right)$ et pour toute fonction quasi-psh $\varphi$ on a $\left\langle\mu_{n}, \varphi\right\rangle \rightarrow\langle\mu, \varphi\rangle$.

Remarque 5.2. Si les $\left(X_{n}, \omega_{n}, f_{n}\right)$ appartiennent à une famille compacte lisse (par exemple une famille finie), les $\mathrm{R}_{n}^{*}$ et $\mathrm{A}_{n}$ sont uniformément bornées (voir remarque 4.5). Dans ce cas, il suffit de supposer que $\delta_{n}\left\|h_{n}\right\|_{\mathrm{L}^{2}(X)}=\mathrm{o}\left(d_{1} \ldots d_{n}\right)$ et que la série $\sum d_{1}^{-1} \ldots d_{n}^{-1} \delta_{n-1}$ converge. Si les $f_{n}$ sont des applications rationnelles dominantes de degré algébrique $s_{n}$ de $\mathbb{P}^{k}$ dans $\mathbb{P}^{k}$, on peut majorer $\delta_{n}$ par $\left(s_{1} \ldots s_{n}\right)^{k-1}$. 
Démonstration. Soit $\varphi$ une fonction quasi-psh sur $X_{0}, \mathrm{dd}^{\mathrm{c}} \varphi \geq-\omega_{0}$. Montrons que $\left\langle\mu_{n}, \varphi\right\rangle$ converge vers une constante $c_{\varphi}$ indépendante de $\left(h_{n}\right)$ (on posera alors $\langle\mu, \varphi\rangle:=c_{\varphi}$ pour $\varphi$ lisse).

Posons $F_{n}:=f_{n} \circ \cdots \circ f_{1}, T_{0}^{-}:=\omega_{0}$ et $T_{0}^{+}:=\mathrm{dd}^{\mathrm{c}} \varphi+\omega_{0}$. Le courant $T_{0}^{+}$est positif fermé et cohomologue à $\omega_{0}$. On définit par récurrence les nombres $b_{n}$ et les fonctions $\varphi_{n}$. Posons

$$
b_{0}:=\int_{X_{0}} \varphi \omega_{0}^{k} \quad \text { et } \quad \varphi_{0}:=\varphi-b_{0} .
$$

D'après les propositions 2.5 et 2.6 , on peut poser

$$
b_{n}:=\int_{X_{n}}\left(f_{n}\right)_{*}\left(\varphi_{n-1}\right) \omega_{n}^{k} \quad \text { et } \quad \varphi_{n}:=\left(f_{n}\right)_{*}\left(\varphi_{n-1}\right)-b_{n} .
$$

On a

$$
\operatorname{dd}^{\mathrm{c}} \varphi_{n}=T_{n}^{+}-T_{n}^{-} \quad \text { avec } T_{n}^{ \pm}=\left(F_{n}\right)_{*}\left(T_{0}^{ \pm}\right)
$$

De plus

$$
\operatorname{cl}\left(T_{n}^{ \pm}\right)=\operatorname{cl}\left(\left(F_{n}\right)_{*}\left(\omega_{0}\right)\right) \quad \text { et } \quad \int_{X_{n}} \varphi_{n} \omega_{n}^{k}=0 .
$$

On en déduit que $\left\|T_{n}^{ \pm}\right\|=\left\|\left(F_{n}\right)_{*}\left(\omega_{0}\right)\right\|=\delta_{n}$.

D'après la proposition 2.2, il existe $\varphi_{n}^{ \pm}$quasi-psh vérifiant $\int_{X_{n}} \varphi_{n}^{ \pm} \omega_{n}^{k}=0$ et

$$
\operatorname{dd}^{\mathrm{c}} \varphi_{n}^{+}-T_{n}^{+}=\operatorname{dd}^{\mathrm{c}} \varphi_{n}^{-}-T_{n}^{-} \geq-r\left(X_{n}, \omega_{n}\right) \delta_{n} \omega_{n} .
$$

On obtient que $\mathrm{dd}^{\mathrm{c}} \varphi_{n}=\mathrm{dd}^{\mathrm{c}}\left(\varphi_{n}^{+}-\varphi_{n}^{-}\right)$. Comme $\int_{X_{n}} \varphi_{n} \omega_{n}^{k}=\int_{X_{n}} \varphi_{n}^{ \pm} \omega_{n}^{k}=0$, on a $\varphi_{n}=\varphi_{n}^{+}-\varphi_{n}^{-}$. Par définition de $\mathrm{A}_{n}$ et $\mathrm{R}_{n}^{*}$, on a

$$
\left|\int_{X_{n}}\left(f_{n}\right)_{*}\left(\varphi_{n-1}^{ \pm}\right) \omega_{n}^{k}\right| \leq \delta_{n-1} \mathrm{~A}_{n} \quad \text { et } \quad\left\|\varphi_{n}^{ \pm}\right\|_{\mathrm{L}^{2}\left(X_{n}\right)} \leq \delta_{n} \mathrm{R}_{n}^{*}
$$

Donc $b_{n} \leq 2 \delta_{n-1} \mathrm{~A}_{n}$ et par hypothèse la série $\sum d_{1}^{-1} \ldots d_{n}^{-1} b_{n}$ converge. Notons $c_{\varphi}$ sa somme. Elle dépend continûment de $\varphi$.

Dans la suite, on intègre seulement sur des ouverts de Zariski convenables car les mesures sont absolument continues par rapport aux mesures de Lebesgue. On a

$$
\begin{aligned}
\left\langle\mu_{n}, \varphi\right\rangle & =\left\langle d_{1}^{-1} \ldots d_{n}^{-1} f_{1}^{*} \ldots f_{n}^{*}\left(v_{n}\right), b_{0}+\varphi_{0}\right\rangle \\
& =b_{0}+\left\langle d_{1}^{-1} \ldots d_{n}^{-1} f_{2}^{*} \ldots f_{n}^{*}\left(v_{n}\right),\left(f_{1}\right)_{*}\left(\varphi_{0}\right)\right\rangle \\
& =b_{0}+\left\langle d_{1}^{-1} \ldots d_{n}^{-1} f_{2}^{*} \ldots f_{n}^{*}\left(v_{n}\right), b_{1}+\varphi_{1}\right\rangle \\
& =b_{0}+d_{1}^{-1} b_{1}+\left\langle d_{1}^{-1} \ldots d_{n}^{-1} f_{2}^{*} \ldots f_{n}^{*}\left(v_{n}\right), \varphi_{1}\right\rangle .
\end{aligned}
$$


Par récurrence, on obtient

$$
\left\langle\mu_{n}, \varphi\right\rangle=b_{0}+d_{1}^{-1} b_{1}+\cdots+d_{1}^{-1} \ldots d_{n}^{-1} b_{n}+d_{1}^{-1} \ldots d_{n}^{-1}\left\langle v_{n}, \varphi_{n}\right\rangle .
$$

Vérifions que $\left\langle\mu_{n}, \varphi\right\rangle$ tend vers $c_{\varphi}$. L'inégalité de Cauchy-Schwarz et les relations (5.1) impliquent que

$$
\begin{aligned}
\left|\left\langle v_{n}, \varphi_{n}\right\rangle\right| & =\left|\left\langle h_{n} \omega_{n}^{k}, \varphi_{n}\right\rangle\right| \leq\left\|h_{n}\right\|_{\mathrm{L}^{2}\left(X_{n}\right)}\left\|\varphi_{n}\right\|_{\mathrm{L}^{2}\left(X_{n}\right)} \\
& \leq\left\|h_{n}\right\|_{\mathrm{L}^{2}\left(X_{n}\right)}\left(\left\|\varphi_{n}^{+}\right\|_{\mathrm{L}^{2}\left(X_{n}\right)}+\left\|\varphi_{n}^{+}\right\|_{\mathrm{L}^{2}\left(X_{n}\right)}\right) \\
& \leq 2\left\|h_{n}\right\|_{\mathrm{L}^{2}\left(X_{n}\right)} \delta_{n} \mathrm{R}_{n}^{*} .
\end{aligned}
$$

La dernière expression est d'ordre o $\left(d_{1} \ldots d_{n}\right)$. Donc $\lim \left\langle\mu_{n}, \varphi\right\rangle=c_{\varphi}$.

Définissons la mesure $\mu$ pour $\varphi$ lisse $\operatorname{par}\langle\mu, \varphi\rangle:=c_{\varphi}$. On a montré que $\mu_{n}$ tend faiblement vers $\mu$. Si $\varphi$ est une fonction quasi-psh quelconque, par semi-continuité supérieure, on a $\langle\mu, \varphi\rangle \geq \lim \sup \left\langle\mu_{n}, \varphi\right\rangle=c_{\varphi}$. D'autre part, comme on peut approcher $\varphi$ par une suite décroissante de fonctions quasi-psh lisses et comme $c_{\varphi}$ dépend continûment de $\varphi$, on a $\langle\mu, \varphi\rangle=c_{\varphi}$ et par suite $\mu$ est PC. De plus, on a $\left\langle\mu_{n}, \varphi\right\rangle \rightarrow\langle\mu, \varphi\rangle$.

Démonstration du théorème 1.3. On montre l'inégalité plus forte suivante

$$
\left|I_{n}(\varphi, \psi)\right| \leq c\left(d_{k-1}+\varepsilon\right)^{n} d_{t}^{-n}\|\varphi\|_{\mathrm{DSH}}\|\psi\|_{\mathrm{L}^{\infty}(\mu)} .
$$

Puisque $\varphi$ s'écrit comme différence de deux fonctions quasi-psh de norme comparable, on peut supposer que $\varphi$ est quasi-psh avec $\operatorname{dd}^{\mathrm{c}} \varphi \geq-\omega$. Du fait que $I_{n}(\varphi, \psi)=$ $-I_{n}(\varphi,-\psi)$, il suffit de majorer $I_{n}(\varphi, \psi)$. Comme $I_{n}(\varphi, \psi+A)=I_{n}(\varphi, \psi)$ pour toute constante $A$, on peut supposer que $\psi$ est positive. On peut également supposer que $\|\psi\|_{\mathrm{L}^{\infty}(\mu)}=1$. Comme $\mu$ est invariante, on a

$$
I_{n}(\varphi, \psi)=\int_{X}\left(d_{t}^{-n}\left(f^{n}\right)_{*}(\varphi)-c_{\varphi}\right) \psi \mathrm{d} \mu \leq\left\|d_{t}^{-n}\left(f^{n}\right)_{*}(\varphi)-c_{\varphi}\right\|_{L^{1}(\mu)} .
$$

On reprend les calculs déjà faits au théorème 5.1. On a

$$
d_{t}^{-n}\left(f^{n}\right)_{*}(\varphi)-c_{\varphi}=d_{t}^{-n} \varphi_{n}^{+}-d_{t}^{-n} \varphi_{n}^{-}-\sum_{i \geq n+1} d_{t}^{-i} b_{i} .
$$

Comme au théorème 5.1, il existe $c_{1}>0$ tel que

$$
\left|b_{i}\right| \leq c_{1}\left(d_{k-1}+\varepsilon\right)^{i-1} \text { et }\left\|\varphi_{n}^{ \pm}\right\|_{L^{2}(X)} \leq c_{1}\left(d_{k-1}+\varepsilon\right)^{n} .
$$

La mesure $\mu$ étant PB, d'après la proposition 2.3, il existe $c_{2}>0$ tel que $\left\|\varphi_{n}^{ \pm}\right\|_{\mathrm{L}^{1}(\mu)} \leq$ $c_{2}\left\|\varphi_{n}^{ \pm}\right\|_{\mathrm{L}^{2}(X)}$. On en déduit que $\left\|d_{t}^{-n}\left(f^{n}\right)_{*}(\varphi)-c_{\varphi}\right\|_{\mathrm{L}^{1}(\mu)} \leq c\left(d_{k-1}+\varepsilon\right)^{n} d_{t}^{-n}, c>0$. Ceci termine la preuve du théorème. 


\section{Distribution des intersections de variétés}

Considérons des TM $F_{i, n}: X \rightarrow X_{i}$ de codimensions $l_{i}, i=1$, 2. On suppose que $l_{1}+l_{2} \geq k$ et que les produits $\Phi_{n}:=F_{1, n} \bullet F_{2, n}$ de $F_{1, n}$ et $F_{2, n}$ sont bien définis. Ce sont des TM de codimension $l_{1}+l_{2}-k$ de $X$ dans $X_{1} \times X_{2}$. Posons $\delta_{i, n}:=\lambda_{k_{i}-1}\left(F_{i, n}\right)$ et $d_{i, n}:=\lambda_{k_{i}}\left(F_{i, n}\right)$.

Théorème 6.1. Supposons que les séries $\sum \delta_{i, n} d_{i, n}^{-1}$ soient convergentes pour $i=1,2$. Alors il existe un sous-ensemble pluripolaire $\&$ de $X_{1} \times X_{2}$ tel que pour tout $\left(x_{1}, x_{2}\right) \in\left(X_{1} \times X_{2}\right) \backslash \mathcal{E}$, la suite de courants

$$
\frac{1}{d_{1, n} d_{2, n}}\left(F_{1, n}^{*}\left(\delta_{x_{1}}\right) \wedge F_{2, n}^{*}\left(\delta_{x_{2}}\right)-F_{1, n}^{*}\left(\omega_{1}^{k_{1}}\right) \wedge F_{2, n}^{*}\left(\omega_{2}^{k_{2}}\right)\right)
$$

tend faiblement vers 0 .

Démonstration. Soit $\delta_{n}$ le degré intermédiaire d'ordre $k_{1}+k_{2}-1$ de $\Phi_{n}$. D'après, la proposition 3.2, il existe une constante $c>0$ telle que

$$
\delta_{n} \leq c\left(\delta_{1, n} d_{2, n}+\delta_{2, n} d_{1, n}\right) .
$$

Le degré intermédiaire d'ordre $k_{1}+k_{2}$ de $\Phi_{n}$ est d'ordre $d_{1, n} d_{2, n}$. Il suffit d'appliquer le théorème 1.1 à $\Phi_{n}$.

Soient $f: X \rightarrow X$ une application biméromorphe et $f^{-1}$ son inverse. Considérons deux familles adaptées régulières $\mathscr{H}^{ \pm}$de sous-ensembles analytiques de dimensions respectives $k-l^{ \pm}$avec $l^{+}+l^{-} \leq k$. Notons $P^{ \pm}: X \rightarrow Y^{ \pm}$les TM associées et $d_{n}^{ \pm}$(resp. $\delta_{n}^{ \pm}$) le degré intermédiaire d'ordre $l^{ \pm}$(resp. d'ordre $l^{ \pm}-1$ ) de $f^{ \pm n}$.

Corollaire 6.2. Supposons que les séries $\sum \delta_{n}^{ \pm}\left[d_{n}^{ \pm}\right]^{-1}$ soient convergentes. Alors il existe un sous-ensemble pluripolaire $\&$ de $Y^{+} \times Y^{-}$tel que pour $\left(a_{1}, b_{1}\right)$ et $\left(a_{2}, b_{2}\right)$ dans $\left(Y^{+} \times Y^{-}\right) \backslash \mathcal{E}$, la suite de courants

$$
\frac{1}{d_{n}^{+} d_{n}^{-}}\left(\left[f^{-n}\left(\mathcal{H}_{a_{1}}^{+}\right) \cap f^{n}\left(\mathcal{H}_{b_{1}}^{-}\right)\right]-\left[f^{-n}\left(\mathcal{H}_{a_{2}}^{+}\right) \cap f^{n}\left(\mathcal{H}_{b_{2}}^{-}\right)\right]\right)
$$

tend faiblement vers 0 .

Démonstration. Posons $F_{n}^{ \pm}:=P^{ \pm} \circ f^{ \pm n}$. Ce sont des TM de codimension $k-l^{ \pm} \mathrm{de}$ $X$ dans $Y^{ \pm}$. D'après la proposition 3.1, ces deux suites de TM vérifient l'hypothèse du théorème 6.1. Il suffit d'appliquer ce théorème.

Nous allons expliciter ce résultat dans le cadre des automorphismes réguliers de $\mathbb{C}^{k}$ introduits par le second auteur [23]. Soit $f$ un automorphisme polynomial de $\mathbb{C}^{k}$. On note aussi $f$ son prolongement comme application birationnelle de $\mathbb{P}^{k}$ dans 
$\mathbb{P}^{k}$. Soit $I^{+}$(resp. $I^{-}$) l'ensemble d'indétermination de $f$ (resp. de $f^{-1}$ ). Ce sont des sous-ensembles analytiques de l'hyperplan à l'infini. L'automorphisme $f$ est dit régulier si $I^{+} \cap I^{-}=\varnothing$. (En dimension 2, les automorphismes réguliers sont ceux du type Hénon). On a alors $\operatorname{dim} I^{+}+\operatorname{dim} I^{-}=k-2$. Posons $s:=\operatorname{dim} I^{-}+1$. Notons $d_{+}$et $d_{-}$les degrés algébriques de $f$ et $f^{-1}$. Ils sont liés par la relation $\left(d_{+}\right)^{s}=\left(d_{-}\right)^{k-s}$.

On peut construire deux courants $T^{ \pm}$positifs fermés de bidegré $(1,1)$ de masse 1 de $\mathbb{P}^{k}$ à potentiel continu dans $\mathbb{C}^{k}$ tels que $f^{*}\left(T^{+}\right)=d_{+} T^{+}, f_{*}\left(T_{-}\right)=d_{-} T^{-}$. Pour $0 \leq l \leq s$ et $0 \leq l^{\prime} \leq k-s$, le courant $T_{l, l^{\prime}}:=\left(T^{+}\right)^{l} \wedge\left(T^{-}\right)^{l^{\prime}}$ est bien défini. Quand $l=s, l^{\prime}=k-s$, on obtient une mesure de probabilité invariante à support compact dans $\mathbb{C}^{k}$. On a également le théorème de convergence suivant :

$$
\lim _{n \rightarrow \infty}\left(d_{+}\right)^{-n l}\left(d_{-}\right)^{-n l^{\prime}}\left(f^{n}\right)^{*}\left(\omega_{\mathrm{FS}}^{l}\right) \wedge\left(f^{n}\right)_{*}\left(\omega_{\mathrm{FS}}^{l^{\prime}}\right)=T_{l, l^{\prime}}
$$

Soient $\mathrm{G}_{l}$ et $\mathrm{G}_{l^{\prime}}$ les grassmanniennes qui paramètrent les sous-espaces projectifs de dimension $k-l$ et $k-l^{\prime}$ de $\mathbb{P}^{k}$. Notons $\mathbb{P}_{x}^{k-l}$ et $\mathbb{P}_{x^{\prime}}^{k-l^{\prime}}$ les sous-espaces projectifs associés aux points $x \in \mathrm{G}_{l}$ et $x^{\prime} \in \mathrm{G}_{l^{\prime}}$.

Théorème 6.3. Soit $f$ un automorphisme régulier de $\mathbb{C}^{k}$ comme ci-dessus. Alors, il existe un sous-ensemble pluripolaire $\mathbb{E}$ de $\mathrm{G}_{l} \times \mathrm{G}_{l^{\prime}}$ tel que pour tout $\left(x, x^{\prime}\right) \in$ $\left(\mathrm{G}_{l} \times \mathrm{G}_{l^{\prime}}\right) \backslash \mathcal{E}$ la suite de courants

$$
\left(d_{+}\right)^{-n l}\left(d_{-}\right)^{-n l^{\prime}}\left[f^{-n}\left(\mathbb{P}_{x}^{k-l}\right) \cap f^{n}\left(\mathbb{P}_{x^{\prime}}^{k-l^{\prime}}\right)\right]
$$

tend faiblement vers le courant invariant $T_{l, l^{\prime}}$.

Démonstration. On prend pour $P^{ \pm}$les TM construites dans l'exemple 3.6(a). Avec les notations du corollaire 6.2 , on a $d_{n}^{+}=d_{+}^{l n}, \delta_{n}^{+}=d_{+}^{(l-1) n}$ et des relations semblables pour les inverses. Il suffit d'appliquer le corollaire 6.2 après avoir intégré par rapport aux variables $a_{2}, b_{2}$. On utilise ensuite la relation (6.1).

\section{Zéros des sections de fibrés en droites}

Soit $X$ une variété projective de dimension $k$ et soit $L$ un fibré en droites ample sur $X$. On munit $L$ d'une métrique hermitienne $h$. Pour toute section holomorphe locale $e_{L}$ de $L$, on définit la norme de $e_{L}$ en chaque point par $\left\|e_{L}\right\|_{h}:=h\left(e_{L}, e_{L}\right)^{1 / 2}$. Soit

$$
c_{1}(h):=-\mathrm{dd}^{\mathrm{c}} \log \left\|e_{L}\right\|_{h}
$$

la forme de courbure de $(L, h)$. Elle représente dans la cohomologie de de Rham la classe de Chern $c_{1}(L) \in H^{2}(X, \mathbb{Z})$ de $L$. Puisque $L$ est ample, on peut choisir $h$ 
de sorte que $c_{1}(h)$ soit une $(1,1)$-forme strictement positive. La variété $X$ est donc munie de la forme de Kähler $\omega:=c_{1}(h)$ et $\int_{X} \omega^{k}=c_{1}(L)^{k} \in \mathbb{Z}^{+}$. Cette intégrale n'est pas égale à 1 en général mais ceci ne changera pas les résultats qui suivent.

Le fibré $L^{n}$ est également muni d'une métrique hermitienne $h_{n}$, induite par la métrique $h$ sur $L$. Plus précisément, $h_{n}$ est définie localement par $\left\|s^{n}\right\|_{h_{n}}=\|s\|_{h}^{n}$. L'espace $\mathrm{H}^{0}\left(X, L^{n}\right)$ des sections holomorphes de $L^{n}$ est muni du produit hermitien naturel

$$
\left\langle s_{1}, s_{2}\right\rangle:=\frac{1}{c_{1}(L)^{k}} \int_{X} h_{n}\left(s_{1}, s_{2}\right) \omega^{k} \quad\left(s_{1}, s_{2} \in \mathrm{H}^{0}\left(M, L^{n}\right)\right) .
$$

Notons $\omega_{\mathrm{FS}}$ la métrique de Fubini-Study de $\mathbb{P} \mathrm{H}^{0}\left(X, L^{n}\right)$. Le lecteur trouvera d'autres notations dans l'exemple 3.6(c). Rappelons que la dimension $k_{n}$ de $\mathbb{P} H^{0}\left(X, L^{n}\right)$ est donnée par le polynôme de Hilbert dont le terme dominant est égal à $c_{1}(L)^{k} n^{k} / k$ ! $[15$, p. 386].

Lemme 7.1. Soient $\delta_{l, n}$ et $d_{l, n}$ les degrés intermédiaires d'ordre $l k_{n}-1$ et d'ordre $l k_{n}$ de $F_{l, n}$. On a $d_{l, n}=n^{l} c_{1}(L)^{k}$ et $\delta_{l, n}=\mathrm{O}\left(n^{l-1}\right)$.

Démonstration. L'invariance des métriques par l'action du groupe unitaire implique que

$$
\Psi_{l, n}^{*} \bar{\Pi}_{l, n}^{*}\left(\omega_{\mathrm{MP}}^{l k_{n}}\right)=\alpha_{l, n} \omega_{\mathrm{FS}}^{l} \quad \text { et } \quad \Psi_{l, n}^{*} \bar{\Pi}_{l, n}^{*}\left(\omega_{\mathrm{MP}}^{l k_{n}-1}\right)=\beta_{l, n} \omega_{\mathrm{FS}}^{l-1}
$$

où $\omega_{\mathrm{MP}}$ est la forme kählérienne naturelle associée à $\mathbb{P}_{l, n}^{X}$ (voir appendice A.3) et $\alpha_{l, n}$, $\beta_{l, n}$ sont des constantes positives. On calcule ces constantes cohomologiquement.

Pour calculer $\alpha_{l, n}$, on remplace $\omega_{\mathrm{MP}}^{l k_{n}}$ dans (7.1) par une masse de Dirac $\delta_{\boldsymbol{s}}$. Son image $\Psi_{l, n}^{*} \bar{\Pi}_{l, n}^{*}\left(\delta_{s}\right)$ sera le courant d'intégration sur un sous-espace projectif de codimension $l$ de $\mathbb{P H}\left(X, L^{n}\right)^{*}$ qui est cohomologue à $\omega_{\mathrm{FS}}^{l}$. Ceci implique que $\alpha_{l, n}=1$.

Soit $T$ le courant d'intégration sur une droite $\mathscr{D} \times\left\{s_{2}\right\} \times \cdots \times\left\{s_{l}\right\}$ de $\mathbb{P}_{l, n}^{X}$. Il est de masse $c_{k_{n}, l}$ (voir appendice A.3). Son image $\Psi_{l, n}^{*} \bar{\Pi}_{l, n}^{*}(T)$ est le courant d'intégration sur un sous-espace de codimension $l-1 \mathrm{de} \mathbb{P H}\left(X, L^{n}\right)^{*}$. La masse de ce dernier courant est égale à 1 . On en déduit que $\beta_{l, n}=c_{k_{n}, l}^{-1}$. En particulier, il est majoré par une constante qui ne dépend que de $l$.

Puisque la classe de $\Phi_{n}^{*}\left(\omega_{\mathrm{FS}}\right)$ est égale à $n c_{1}(L)$, on a

$$
d_{l, n}=\int_{X} \Phi_{n}^{*} \Psi_{n}^{*} \bar{\Pi}_{l, n}^{*}\left(\omega_{\mathrm{MP}}^{l k_{n}}\right) \wedge \omega^{k-l}=\int_{X} \Phi_{n}^{*}\left(\omega_{\mathrm{FS}}^{l}\right) \wedge \omega^{k-l}=n^{l} c_{1}(L)^{k}
$$

et

$$
\begin{aligned}
\delta_{l, n} & =\int_{X} \Phi_{n}^{*} \Psi_{n}^{*} \bar{\Pi}_{l, n}^{*}\left(\omega_{\mathrm{MP}}^{l k_{n}-1}\right) \wedge \omega^{k-l+1}=\int_{X} \beta_{l, n} \Phi_{n}^{*}\left(\omega_{\mathrm{FS}}^{l-1}\right) \wedge \omega^{k-l+1} \\
& =\beta_{l, n} n^{l-1} c_{1}(L)^{k}
\end{aligned}
$$


Vol. 81 (2006) Distribution des valeurs de transformations méromorphes et applications 249

On voit que la série $\sum \delta_{l, n} d_{l, n}^{-1}$ ne converge pas. C'est donc le théorème 4.1(2) que nous appliquerons. Le théorème suivant, dû à Zelditch [26], est une amélioration d'un théorème de Tian [24]. Il donne la convergence en moyenne des courants $n^{-l}\left[\mathbf{Z}_{\boldsymbol{s}_{n}}\right]$ avec $\boldsymbol{s}_{n} \in \mathbb{P}_{l, n}^{X}$.

Théorème 7.2 ([26]). Pour tout $r \geq 0$, on $a$

$$
\left\|n^{-l} \Phi_{n}^{*}\left(\omega_{\mathrm{FS}}^{l}\right)-\omega^{l}\right\|_{\mathrm{e}^{r}}=\mathrm{O}\left(n^{-1}\right)
$$

Soient $\sigma_{n}$ des mesures de probabilité $\mathrm{PB} \operatorname{sur} \mathbb{P}_{l, n}^{X}$. Posons $\mathrm{R}_{i, n}:=\mathrm{R}_{i}\left(\mathbb{P}_{l, n}^{X}, \omega_{\mathrm{MP}}, \sigma_{n}\right)$ et $\Delta_{n}(t):=\Delta\left(\mathbb{P}_{l, n}^{X}, \omega_{\mathrm{MP}}, \sigma_{n}, t\right)$. On munit $\mathbb{P}_{l}^{X}:=\prod_{n \geq 1} \mathbb{P}_{l, n}^{X}$ de la mesure $\sigma$, produit $\operatorname{des} \sigma_{n}$. Faisons des hypothèses sur les mesures $\sigma_{n}$.

Théorème 7.3. Supposons que la série $\sum_{n \geq 1} \Delta_{n}(n t)$ converge pour tout $t>0$. Supposons aussi que $\mathrm{R}_{1, n}=\mathrm{o}(n)$ et $\mathrm{R}_{3, n}=\mathrm{o}(n)$. Alors pour $\sigma$-presque tout $\mathbf{s}=$ $\left(\boldsymbol{s}_{n}\right) \in \mathbb{P}_{l}^{X}$, la suite de courants $n^{-l}\left[\mathbf{Z}_{\boldsymbol{s}_{n}}\right]$ tend faiblement vers $\omega^{l}$.

Démonstration. Les relations (7.1) et le théorème 7.2 entraînent que $n^{-l} F_{l, n}^{*}\left(\omega_{\mathrm{MP}}^{l k_{n}}\right)$ tend vers $\omega^{l}$ dans $\mathcal{C}^{r}$ pour tout $r \geq 0$. D'après la proposition $4.3, n^{-l} F_{l, n}^{*}\left(\sigma_{n}\right)$ tend faiblement vers $\omega^{l}$ car $n^{-l} \delta_{l, n} \mathrm{R}_{3, n}$ tend vers 0 par hypothèse. D'après le théorème 4.1(2), pour $\sigma$-presque tout $\boldsymbol{s} \in \mathbb{P}_{l}^{X}, n^{-l}\left(\left[\mathbf{Z}_{\boldsymbol{s}_{n}}\right]-F_{l, n}^{*}\left(\sigma_{n}\right)\right)$ tend faiblement vers 0 . Le théorème en découle.

Posons $\mathrm{G}_{l}^{X}:=\prod_{n \geq 1} \mathrm{G}_{l, n}^{X}$. Soit $\Omega_{l, n}$ la mesure de probabilité invariante sur $\mathrm{G}_{l, n}^{X}$. Notons $\Omega_{l}$ la mesure de probabilité sur $\mathrm{G}_{l}^{X}$, produit des $\Omega_{l, n}$. Fixons des sous-espaces réels $\mathbb{R} \mathbb{P} H^{0}\left(X, L^{n}\right)$ de $\mathbb{P H}^{0}\left(X, L^{n}\right)$ invariants par l'action du groupe orthogonal associé. Soit $\mathbb{R G}_{l, n}^{X}$ la sous-grassmannienne totalement réelle de $\mathrm{G}_{l, n}^{X}$ correspondante. Soit $m_{l, n}$ la mesure invariante de masse 1 sur $\mathbb{R G}_{l, n}^{X}$. Notons $m_{l}$ le produit des $m_{l, n}$ qui est une mesure de probabilité sur $\mathrm{G}_{l}^{X}$.

Corollaire 7.4. Soient $\mu_{l, n}:=\Omega_{l, n}$ et $\mu_{l}:=\Omega_{l}\left(\right.$ ou $\mu_{l, n}:=m_{l, n}$ et $\left.\mu_{l}:=m_{l}\right)$. Alors pour $\mu_{l}$-presque tout $\check{\boldsymbol{s}}=\left(\check{\boldsymbol{s}}_{n}\right) \in \mathrm{G}_{l}^{X}$, la suite de courants $n^{-l}\left[\mathbf{Z}_{\check{\boldsymbol{s}}_{n}}\right]$ tend faiblement vers $\omega^{l}$. De plus, si on pose

$$
E_{l, n}(\varepsilon):=\bigcup_{\|\psi\|_{\mathcal{C}^{2}} \leq 1}\left\{\check{\boldsymbol{s}}_{n} \in \mathrm{G}_{l, n}^{X}, \quad\left|\left\langle n^{-l}\left[\mathbf{Z}_{\check{\boldsymbol{s}}_{n}}\right]-\omega^{l}, \psi\right\rangle\right| \geq \varepsilon\right\}
$$

on $a$

$$
\mu_{l, n}\left(E_{l, n}(\varepsilon)\right) \leq c n^{m k} \exp (-\alpha \varepsilon n)
$$

où $m \geq 0, \alpha>0$ et $c>0$ sont des constantes indépendantes de $\varepsilon$. 
Démonstration. Notons $\mathbb{R} \mathbb{P}_{l, n}^{X}:=\mathbb{R P} \mathrm{H}^{0}\left(X, L^{n}\right) \times \cdots \times \mathbb{R} \mathbb{P} \mathrm{H}^{0}\left(X, L^{n}\right)(l$ fois $)$. Soient $\widetilde{\Omega}_{l, n}$ (resp. $\left.\tilde{m}_{l, n}\right)$ la mesure de probabilité invariante naturelle $\operatorname{sur} \mathbb{P}_{k, l}^{X}$ (resp. sur $\mathbb{R P}_{l, n}^{X}$ ) (voir appendice A.3) et $\widetilde{\Omega}_{l}$ (resp. $\widetilde{m}_{l}$ ) la mesure de probabilité $\operatorname{sur} \mathbb{P}_{l}^{X}$, produit des $\widetilde{\Omega}_{l, n}$ (resp. des $\left.\widetilde{m}_{l, n}\right)$. L'invariance des mesures considérées implique que $\bar{\Pi}_{l, n}^{*}\left(\widetilde{\Omega}_{l, n}\right)=$ $\Omega_{l, n}$ et $\bar{\Pi}_{l, n}^{*}\left(\tilde{m}_{l, n}\right)=m_{l, n}$.

Soient $\tilde{\mu}_{l, n}=\widetilde{\Omega}_{l, n}$ ou $\tilde{m}_{l, n}$ et $\tilde{\mu}_{l}=\tilde{\Omega}_{l}$ ou $\tilde{m}_{l}$. Il suffit de montrer que pour $\tilde{\mu}$-presque tout $\boldsymbol{s}=\left(\boldsymbol{s}_{1}, \boldsymbol{s}_{2}, \ldots\right) \in \mathbb{P}_{l}^{X}$ on a $n^{-l}\left[\mathbf{Z}_{\boldsymbol{s}_{n}}\right] \rightarrow \omega^{l}$ et

$$
\tilde{\mu}_{l, n}\left(\Pi_{l, n}^{-1}\left(E_{l, n}(\varepsilon)\right)\right) \leq c n^{m k} \exp (-\alpha \varepsilon n) .
$$

Cela résulte des théorèmes $7.2,7.3$, la proposition $4.2(\mathrm{c})$ et la première inégalité dans (4.4). Les estimations sur $\mathrm{R}_{i, n}$ et $\Delta_{n}$ sont fournies par la proposition A.9. La dimension de $\mathbb{P}_{l, n}^{X}$ est de l'ordre $n^{k}$.

Remarques 7.5. Soit $\left(c_{n}\right)$ une suite de nombre réels positifs vérifiant $c_{n}=\mathrm{o}(n / \log n)$.

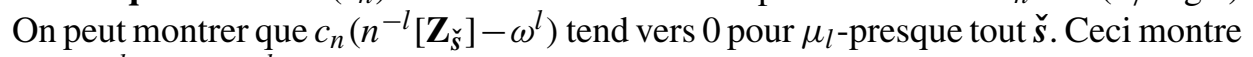
que $n^{-l}\left[\mathbf{Z}_{\breve{s}}\right]-\omega^{l}$ tend vers 0 à vitesse $\simeq \log n / n$. Obsevons que la multiplication par $c_{n}$ revient à diviser $\varepsilon$ par $c_{n}$. On obtient aussi des convergences avec estimation lorsqu'on teste les formes höldériennes (voir remarque 1.4). Dans le cas où $l=1$, Shiffman et Zelditch [22] ont démontré la convergence $n^{-1}\left[\mathbf{Z}_{s_{n}}\right] \rightarrow \omega$ pour $\Omega_{1^{-}}$ presque toute suite $s=\left(s_{n}\right), s_{n} \in \mathbb{P} \mathrm{H}^{0}\left(X, L^{n}\right)$. Ils ont prouvé que la vitesse de convergence est majorée par $n^{\varepsilon-1 / 2}$ pour les observables $\mathcal{C}^{2}$.

\section{A. Estimations des constantes}

A.1. Ensembles pluripolaires, capacités, mesures modérées. Josefson [11] a montré qu'un sous-ensemble localement pluripolaire dans $\mathbb{C}^{k}$ est pluripolaire. Alexander a étendu ce résultat à $\mathbb{P}^{k}[1]$. Il en résulte que pour toute variété projective $X$ de dimension $k$, un ensemble $E$ localement pluripolaire l'est globalement : il suffit d'utiliser une application holomorphe finie $\pi: X \rightarrow \mathbb{P}^{k}$. Si $\varphi$ est quasi-psh telle que $\pi(E) \subset(\varphi=-\infty)$, on a $E \subset\left(\pi^{*} \varphi=-\infty\right)$. Il serait utile de montrer ce résultat pour toute variété complexe compacte. On utilise à plusieurs reprises le résultat suivant.

Proposition A.1. Soit $(X, \omega)$ une variété kählérienne compacte de dimension $k$. Tout sous-ensemble analytique propre $Y \subset X$ est pluripolaire.

Démonstration. Si $Y$ est une hypersurface de $X$, il existe $\varphi$ quasi-psh telle que $\mathrm{dd}^{\mathrm{c}} \varphi=$ $[Y]-\alpha$ avec $\alpha$ lisse. Il est clair que $Y=(\varphi=-\infty)$ et $\varphi$ est lisse sur $X \backslash Y$. Si $\operatorname{dim} Y<k-1$, on construit une variété kählérienne $\widehat{X}$ par des éclatements successifs 
Vol. 81 (2006) Distribution des valeurs de transformations méromorphes et applications 251

le long $Y$ ou ses singularités. Si $\pi$ désigne la projection de $\widehat{X}$ sur $X, \pi^{-1}(Y)$ est une hypersurface de $\widehat{X}$. Soit $\psi$ quasi-psh sur $\widehat{X}$, lisse sur $\widehat{X} \backslash \pi^{-1}(Y)$ telle que $\pi^{-1}(Y)=(\psi=-\infty)$. Puisque $\mathrm{dd}^{\mathrm{c}} \psi$ s'écrit comme différence de deux courants positifs fermés, lisses sur $\widehat{X} \backslash \pi^{-1}(Y)$, on a $\pi_{*} \psi=u_{1}-u_{2}$ avec $u_{i}$ quasi-psh lisses sur $X \backslash Y$. Puisque $\pi_{*} \psi(x)$ tend vers $-\infty$ quand $x$ tend vers $Y$, on a $Y \subset\left(u_{1}=-\infty\right)$ car $u_{2}$ est bornée supérieurement.

Introduisons une capacité dont les ensembles de capacité nulle sont les ensembles pluripolaires. Cela a été fait par Alexander pour $\mathbb{P}^{k}$. Notons $\pi: \mathbb{C}^{k+1} \backslash\{0\} \rightarrow \mathbb{P}^{k}$ la projection canonique. Soient $\delta^{2 k+1}$ la sphère unité de $\mathbb{C}^{k+1}$ et $\sigma_{2 k+1}$ la mesure de probabilité invariante sur $8^{2 k+1}$. Alexander a posé pour un sous-ensemble $K$ de $\mathbb{P}^{k}$ :

$$
\operatorname{cap}^{\prime}(K):=\inf _{f} \sup _{\pi^{-1}(K) \cap 8^{2 k+1}}|f|^{1 / n}
$$

où $f$ parcourt les polynômes homogènes de degré $n$ de $\mathbb{C}^{k+1}$ vérifiant

$$
\int_{\delta^{2 k+1}}\left(\log |f|^{1 / n}-\log \left|z_{1}\right|\right) \mathrm{d} \sigma_{2 k+1}=0 .
$$

Etant donné un sous-ensemble $K$ d'une variété kählérienne compacte $(X, \omega)$ nous définissons la capacité de $K$ par

$$
\operatorname{cap}(K):=\inf _{\varphi}\left\{\exp \left(\sup _{K} \varphi\right), \varphi \text { quasi-psh, } \mathrm{dd}^{\mathrm{c}} \varphi \geq-\omega, \max _{X} \varphi=0\right\} .
$$

Dans $\mathbb{P}^{k}$ toute fonction quasi-psh $\varphi$, vérifiant $\mathrm{dd}^{\mathrm{c}} \varphi \geq-\omega_{\mathrm{FS}}$, est limite de fonctions sur $\mathbb{P}^{k}$ de la forme $\log |f|^{1 / n}-\log \|z\|$ où $f$ est homogène de degré $n$. En utilisant la proposition A.3 ci-dessous on peut montrer que

$$
\operatorname{cap}^{\prime}(K) \leq \operatorname{cap}(K) \leq \sqrt{k \mathrm{e}} \operatorname{cap}^{\prime}(K) .
$$

Dans la suite nous utilisons la capacité cap qui a un sens pour toute variété kählérienne compacte. Avec notre normalisation, on a toujours $\operatorname{cap}(X)=1$. On vérifie que $\operatorname{cap}(K)=0$ si et seulement si $K$ est pluripolaire.

Proposition A.2. Soit $\sigma$ la mesure associée à la forme volume $\omega^{k}$. Alors, il existe $c>0$ et $\alpha>0$ tels que $\sigma$ soit $(c, \alpha)$-modérée. En particulier, on a $\Delta(X, \omega, \sigma, t) \leq$ $c \exp \left(-\alpha r^{-1} t\right)$ pour tout $t \in \mathbb{R}$, où $r:=r(X, \omega)$.

Démonstration. Notons $\mathrm{B}(a, r)$ (resp. $\left.\mathrm{B}_{r}\right)$ la boule de $\mathbb{C}^{k}$ de rayon $r$ centrée en $a$ (resp. centrée en 0 ). Posons $\omega_{0}:=\mathrm{dd}^{\mathrm{c}}\|z\|^{2}$ la forme euclidienne sur $\mathbb{C}^{k}$ et $\sigma_{0}:=\omega_{0}^{k}$. Soit $\Psi_{n}: \mathrm{B}_{4} \rightarrow X$ une famille finie d'applications holomorphes injectives telles que les ouverts $\Psi_{n}\left(\mathrm{~B}_{1}\right)$ recouvrent $X$. Soit $A_{1}>0$ tel que pour tout $n, \Psi_{n}^{*}(\omega) \leq A_{1} \omega_{0}$ et $\Psi_{n}^{*}(\sigma) \leq A_{1} \sigma_{0}$ sur $\mathrm{B}_{3}$. 
Soit $\varphi$ telle que $\operatorname{dd}^{\mathrm{c}} \varphi \geq-\omega$ et $\int_{X} \varphi \omega^{k}=0$. Posons $\varphi_{n}:=\varphi \circ \Psi_{n}$. Il suffit de montrer que $\int_{\mathrm{B}_{1}} \exp \left(-\alpha^{\prime} \varphi_{n}\right) \omega_{0}^{k} \leq c^{\prime}$ pour $c^{\prime}>0, \alpha^{\prime}>0$ indépendants de $\varphi$. D'après la proposition 2.1, il existe $A_{2}>0$ tel que $\varphi \leq A_{2}$ et $\int|\varphi| \mathrm{d} \sigma \leq A_{2}$. Ceci implique que $\sigma(\varphi<-M) \leq A_{2} M^{-1}$ pour tout $M>0$. Soit $M>0$ tel que $A_{2} M^{-1}<\sigma\left(\Psi_{n}\left(\mathrm{~B}_{1}\right)\right)$ pour tout $n$. La dernière relation implique que $(\varphi<-M)$ ne peut contenir $\Psi_{n}\left(\mathrm{~B}_{1}\right)$. On peut donc choisir un point $a_{n} \in \mathrm{B}_{1}$ tel que $\varphi_{n}\left(a_{n}\right)=\varphi\left(\Phi_{n}\left(a_{n}\right)\right) \geq-M$.

Posons $\psi_{n}:=\varphi_{n}+A_{1}\left(\|z\|^{2}-16\right)$. C'est une fonction psh dans $\mathrm{B}_{3}$ vérifiant $\psi_{n} \leq$ $\varphi_{n}$. Montrons que $\int_{\mathrm{B}\left(a_{n}, 2\right)} \exp \left(-\alpha^{\prime} \psi_{n}\right) \omega_{0}^{k} \leq c^{\prime}$ pour $c^{\prime}>0$ et $\alpha^{\prime}>0$ convenables. On a dd $\psi_{n} \geq 0, \psi_{n}\left(a_{n}\right) \geq-M-16 A_{1}$ et $\psi_{n} \leq A_{2}$ sur $\mathrm{B}\left(a_{n}, 2\right) \subset \mathrm{B}_{4}$. Il suffit d'appliquer un théorème de Hörmander [14, p. 97] qui affirme que $\int_{\mathrm{B}_{1}} \exp (-\phi) \omega_{0}^{k} \leq$ $c^{\prime}$ pour toute fonction psh $\phi$ sur $\mathrm{B}_{2}$ avec $\phi(0)=0$ et $\phi \leq 1$. On peut prendre $\alpha^{\prime}=\left(A_{2}+16 A_{1}+M\right)^{-1}$.

A.2. Estimation des constantes pour $\mathbb{P}^{\boldsymbol{k}}$. Notons $\delta^{k}$ (resp. $\delta^{2 k+1}$ ) la sphère unité de $\mathbb{R}^{k+1}$ (resp. de $\mathbb{C}^{k+1}$ ) et $\sigma_{k}$ (resp. $\sigma_{2 k+1}$ ) la mesure invariante de masse 1 sur $\delta^{k}$ (resp. $\left.8^{2 k+1}\right)$. Soit $\pi: \mathbb{C}^{k+1} \backslash\{0\} \rightarrow \mathbb{P}^{k}$ la projection canonique. Soient $z=\left(z_{0}, \ldots, z_{k}\right)$ les coordonnées de $\mathbb{C}^{k+1}$. On dira qu'une fonction $\Phi$ sur $\mathbb{C}^{k+1}$ est log-homogène si $\Phi(\lambda z)=\log |\lambda|+\Phi(z)$ pour tout $\lambda \in \mathbb{C}^{*}$. La fonction log $\|z\|$ est psh log-homogène et $\pi^{*}\left(\omega_{\mathrm{FS}}\right)=\mathrm{dd}^{\mathrm{c}} \log \|z\|$.

Notons $\Omega_{\mathrm{FS}}$ la mesure sur $\mathbb{P}^{k}$ associée à la forme volume $\omega_{\mathrm{FS}}^{k}$. C'est la mesure invariante de masse 1 sur $\mathbb{P}^{k}$. Soit $\varphi$ une fonction quasi-psh vérifiant $\operatorname{dd}^{\mathrm{c}} \varphi \geq-\omega_{\mathrm{FS}}$. Posons $\Phi:=\varphi \circ \pi+\log \|z\|$ et $\Phi(0):=-\infty$. C'est une fonction psh log-homogène sur $\mathbb{C}^{k+1}$ vérifiant $\max _{\S^{2 k+1}} \Phi=\max _{\mathbb{P}^{k}} \varphi$ et $\int_{\S^{2 k+1}} \Phi \mathrm{d} \sigma_{2 k+1}=\int_{\mathbb{P}^{k}} \varphi \omega_{\mathrm{FS}}^{k}$. Soit $\mathbb{R}^{k}$ l'image de $\mathbb{R}^{k+1}$ par $\pi$. C'est un sous-espace projectif réel de dimension $k$ de $\mathbb{P}^{k}$. Posons $m_{\mathrm{FS}}:=\pi_{*}\left(\sigma_{k}\right)$. On a aussi $\max _{g^{k}} \Phi=\max _{\mathbb{R}^{k}} \varphi$ et $\int_{\xi^{k}} \Phi \mathrm{d} \sigma_{k}=\int \varphi \mathrm{d} m_{\mathrm{FS}}$.

Proposition A.3. On a

$$
\mathrm{R}_{1}^{*}\left(\mathbb{P}^{k}, \omega_{\mathrm{FS}}\right) \leq \frac{1}{2}(1+\log k) \quad \text { et } \quad \mathrm{R}_{2}^{*}\left(\mathbb{P}^{k}, \omega_{\mathrm{FS}}, 1\right) \leq 1+\log k .
$$

Démonstration. Rappelons que $r\left(\mathbb{P}^{k}, \omega_{\mathrm{FS}}\right)=1$. Soit $m:=\max _{\mathbb{P}^{k}} \varphi$. D'après Alexander [1, Theorem 2.2], on a

$$
\begin{aligned}
\int_{\delta^{2 k+1}} \Phi \mathrm{d} \sigma_{2 k+1} & \geq m+\int_{\delta^{2 k+1}} \log \left|z_{1}\right| \mathrm{d} \sigma_{2 k+1} \\
& =m-\frac{1}{2} \sum_{n=1}^{k} \frac{1}{n} \geq m-\frac{1}{2}(1+\log k) .
\end{aligned}
$$

On en déduit que $m \leq \frac{1}{2}(1+\log k)$ si $\int_{\mathbb{P}^{k}} \varphi \omega_{\mathrm{FS}}^{k}=0$. $\mathrm{D}^{\prime}$ où $\mathrm{R}_{1}^{*}\left(\mathbb{P}^{k}, \omega_{\mathrm{FS}}\right) \leq \frac{1}{2}(1+\log k)$. D'après la proposition 2.4 , on a $\mathrm{R}_{2}^{*}\left(\mathbb{P}^{k}, \omega_{\mathrm{FS}}, 1\right) \leq 1+\log k$. 
Vol. 81 (2006) Distribution des valeurs de transformations méromorphes et applications 253

La proposition suivante permet d'estimer les intégrales sur $8^{k}$ en fonction d'intégrales sur des sous-espaces linéaires. Sa démonstration résulte du théorème du Fubini en écrivant les mesures $\sigma_{m}$ en coordonnées polaires.

Proposition A.4. Soit h une fonction mesurable positive sur la sphère unité $8^{k}$ de $\mathbb{R}^{k+1}$ (resp. $\delta^{2 k+1}$ de $\mathbb{C}^{k+1}$ ). Soit $F$ un sous-espace réel (resp. complexe) de dimension $m$ de $\mathbb{R}^{k+1}$ (resp. de $\mathbb{C}^{k+1}$ ), $1 \leq m \leq k$. Supposons que pour tout sous-espace réel (resp. complexe) E de dimension $m+1$ contenant $F$ on ait $\int_{\delta^{k} \cap E} h \mathrm{~d} \sigma_{m} \leq A$ (resp. $\int_{8^{2 k+1} \cap E} h \mathrm{~d} \sigma_{2 m+1} \leq A$ ) où $A>0$ est une constante. Alors il existe une constante $c>0$ indépendante de $k, A$ et $h$ telle que $\int_{g^{k}} h \mathrm{~d} \sigma_{k} \leq c A k^{m / 2}$ (resp. $\left.\int_{\S^{2 k+1}} h \mathrm{~d} \sigma_{2 k+1} \leq c A k^{m}\right)$.

Dans le corollaire suivant, on prend $m=1$ et la droite $F$ associée à un point $z$ $\operatorname{avec} \varphi(z)=0$.

Corollaire A.5. Il existe $c>0$ et $\alpha>0$ indépendants de $k$, tels que pour toute $\varphi$ quasi-psh vérifiant $\mathrm{dd}^{\mathrm{c}} \varphi \geq-\omega_{\mathrm{FS}}$ et $\max _{\mathbb{P}^{k}} \varphi=0$ on a $\int_{\mathbb{P}^{k}} \exp (-\alpha \varphi) \omega_{\mathrm{FS}}^{k} \leq c k$. En particulier, on a $\Delta\left(\mathbb{P}^{k}, \omega_{\mathrm{FS}}, \Omega_{\mathrm{FS}}, t\right) \leq$ ck $\exp (-\alpha t)$ pour tout $t \in \mathbb{R}$.

La proposition suivante explique pourquoi les estimations pour $\mathbb{R} \mathbb{P}^{k}$ et $\mathbb{P}^{k}$ sont essentiellement les mêmes. Rappelons que dans $\mathbb{P}^{k}$ on a cap $\left(\mathbb{P}^{k}\right)=1$.

Proposition A.6. Soit cap $\left(\mathbb{R} \mathbb{P}^{k}\right)$ la capacité de $\mathbb{R} \mathbb{P}^{k}$ dans $\mathbb{P}^{k}$. Alors pour tout $k \geq 2$ et toute fonction $\varphi$ quasi-psh sur $\mathbb{P}^{k}$ vérifiant $\operatorname{dd}^{\mathrm{c}} \varphi \geq-\omega_{\mathrm{FS}}$ et $\max _{\mathbb{P}^{k}} \varphi=0$, on a $\max _{\mathbb{R P}^{k}} \varphi \geq \log \operatorname{cap}\left(\mathbb{R P}^{2}\right)$. En particulier, $\operatorname{cap}\left(\mathbb{R}^{k}\right)=\operatorname{cap}\left(\mathbb{R} \mathbb{P}^{2}\right)$ pour tout $k \geq 2$.

Démonstration. Soient $a \in \mathbb{P}^{k}$ et $b \in \mathbb{R}^{k}$ tels que $\varphi(a)=0$ et $\varphi(b)=\max _{\mathbb{R} \mathbb{P}^{k}} \varphi$. Observons que $\mathbb{C}^{k+1}$ est réunion des sous-espaces complexes $E$ de dimension 3 contenant une droite réelle fixée de $\mathbb{R}^{k+1}$ et vérifiant $\operatorname{dim}_{\mathbb{R}}\left(E \cap \mathbb{R}^{k+1}\right)=3$. Donc $\mathbb{P}^{k}$ est réunion des plans projectifs $P$ de $\mathbb{P}^{k}$ passant par $b$ et vérifiant $\operatorname{dim}_{\mathbb{R}}\left(P \cap \mathbb{R} \mathbb{P}^{k}\right)=2$. Soit $P$ un tel plan contenant $a$. Par définition de la capacité sur $P \simeq \mathbb{P}^{2}$ pour $P \cap \mathbb{R} \mathbb{P}^{k} \simeq \mathbb{R}^{2}$, on a $\varphi(b) \geq \log \operatorname{cap}\left(\mathbb{R P}^{2}\right)$. Donc $\operatorname{cap}\left(\mathbb{R} \mathbb{P}^{k}\right) \geq \operatorname{cap}\left(\mathbb{R} \mathbb{P}^{2}\right)$. On obtient l'autre inégalité en observant que toute fonction quasi-psh $\varphi$ sur un plan $P \simeq \mathbb{P}^{2}$ de $\mathbb{P}^{k}$ avec dd $\mathrm{dd}^{\mathrm{c}} \varphi \geq-\omega_{\mathrm{FS}}$ se prolonge en fonction quasi-psh $\widetilde{\varphi}$ sur $\mathbb{P}^{k}$ avec $\operatorname{dd}^{\mathrm{c}} \widetilde{\varphi} \geq-\omega_{\mathrm{FS}}$ et $\max _{\mathbb{P} k} \widetilde{\varphi}=\max _{\mathbb{P}^{2}} \varphi$ (ceci se voit aisément sur le relevé de $\left.\widetilde{\varphi} \mathrm{a} \mathbb{C}^{k+1}\right)$.

Proposition A.7. Il existe des constantes $c>0$ et $\alpha>0$ indépendantes de $k$ telles qu'on ait $\int_{\mathbb{P} k} \exp (-\alpha \varphi) \mathrm{d} m_{\mathrm{FS}} \leq c \sqrt{k}$ pour $\varphi$ comme ci-dessus, $\mathrm{R}_{i}\left(\mathbb{P}^{k}, \omega_{\mathrm{FS}}, m_{\mathrm{FS}}\right) \leq$ $c(1+\log k)$ pour $i=1,2,3$ et $\Delta\left(\mathbb{P}^{k}, \omega_{\mathrm{FS}}, m_{\mathrm{FS}}, t\right) \leq c \sqrt{k} \exp (-\alpha t)$ pour tout $t \in \mathbb{R}$. 
Démonstration. On peut reprendre la démonstration de Hörmander [14, p. 98] pour les fonctions $\psi$ sous-harmoniques sur le disque unité de $\mathbb{C}$ qui vérifient $\psi(0)=0$ et $\psi \leq 1$. On remplace la mesure de Lebesgue par la mesure $m$ sur le cercle $|z|=1 / 2$. On trouve $\int \exp (-\psi / 2) \mathrm{d} m \leq c_{1}$ avec $c_{1}>0$ indépendant de $\psi$. En utilisant un recouvrement comme dans la proposition A.2, on montre qu'il existe $c^{\prime}>0$ et $\alpha>0$ tels que $\int \exp \left(-\alpha^{\prime} \psi\right) \mathrm{d} m_{\mathrm{FS}} \leq c^{\prime}$ pour $\psi$ quasi-sh sur $\mathbb{P}^{1}$ vérifiant $\mathrm{dd}^{\mathrm{c}} \psi \geq-\omega_{\mathrm{FS}}$ et $\max _{\mathbb{P}^{1}} \psi=0$. Le passage à $\mathbb{P}^{k}$ avec l'estimation $\int_{\mathbb{P}^{k}} \exp (-\alpha \varphi) \mathrm{d} m_{\mathrm{FS}} \leq c \sqrt{k}$ est une simple application des propositions A.4 et A.6. On en déduit que $\Delta\left(\mathbb{P}^{k}, \omega_{\mathrm{FS}}, m_{\mathrm{FS}}, t\right) \leq$ $c \sqrt{k} \exp (-\alpha t)$.

Montrons que $\mathrm{R}_{1}\left(\mathbb{P}^{k}, \omega_{\mathrm{FS}}, m_{k}\right) \leq c(1+\log k)$. D'après (2.4), il suffit de vérifier que $-\int \varphi \mathrm{d} m_{\mathrm{FS}} \leq c(1+\log k)$. On peut supposer que $k \geq 2$. D'après l'estimation $\int_{\mathbb{P}^{k}} \exp (-\alpha \varphi) \mathrm{d} m_{\mathrm{FS}} \leq c \sqrt{k}$ ci-dessus on a pour tout $t_{0} \geq 0$

$$
\begin{aligned}
-\int \varphi \mathrm{d} m_{\mathrm{FS}} & \leq t_{0}+\int_{t_{0}}^{+\infty} m_{\mathrm{FS}}(\varphi<-t) \mathrm{d} t \leq t_{0}+\int_{t_{0}}^{+\infty} c \sqrt{k} \exp (-\alpha t) \mathrm{d} t \\
& =t_{0}+c \sqrt{k} \alpha^{-1} \exp \left(-\alpha t_{0}\right) .
\end{aligned}
$$

Pour $t_{0}=2^{-1} \alpha^{-1} \log k$, on obtient l'inégalité voulue.

D'après les propositions 2.4 et $\mathrm{A} .3$, on a $\mathrm{R}_{i}\left(\mathbb{P}^{k}, \omega_{\mathrm{FS}}, m_{\mathrm{FS}}\right) \leq c(1+\log k)$ pour $i=2,3$ avec $c>0$ convenable.

A.3. Espaces produits et espaces multiprojectifs. On associe à la variété $X:=$ $X_{1} \times X_{2}$ la forme de Kähler

$$
\omega:=c_{12}\left(\pi_{1}^{*} \omega_{1}+\pi_{2}^{*} \omega_{2}\right)
$$

où les $\pi_{i}$ sont les projections canoniques de $X$ sur $X_{i}$ et où $c_{12}>0$ est tel que $\int_{X} \omega^{k_{1}+k_{2}}=1$. La constante $c_{12}$ est calculée par la formule

$$
c_{12}^{-k_{1}-k_{2}}=\left(\begin{array}{c}
k_{1}+k_{2} \\
k_{1}
\end{array}\right) .
$$

Considérons deux mesures de probabilité $\mu_{i}$ sur $X_{i}$ et $\mu$ le produit de $\mu_{1}$ et $\mu_{2}$. C'est une mesure de probabilité sur $X$. Posons $r:=r(X, \omega)$.

Proposition A.8. Soit $\mathrm{R}$ une constante vérifiant $\mathrm{R} \geq \mathrm{R}_{1}\left(X_{i}, \omega_{i}, \mu_{i}\right)$ pour $i=1,2$. Supposons qu'il existe $c>0$ et $\alpha>0$ tels que $\Delta\left(X_{i}, \omega_{i}, \mu_{i}, t\right) \leq c \exp (-\alpha t)$ pour $t \in \mathbb{R}$ et $i=1,2$. Alors on a

$$
\mathrm{R}_{1}(X, \omega, \mu) \leq 2 r \mathrm{R}+2 r \alpha^{-1} \log c+4 \alpha^{-1} r
$$

et pour tout $t \in \mathbb{R}$

$$
\Delta(X, \omega, \mu, t) \leq 2 c \exp (\alpha \mathrm{R}) \exp \left(-\alpha r^{-1} t / 2\right) .
$$


Vol. 81 (2006) Distribution des valeurs de transformations méromorphes et applications 255

Démonstration. Fixons une fonction $\psi$ sur $X=X_{1} \times X_{2}$ telle que $\max _{X} \psi=0$ et $\operatorname{dd}^{\mathrm{c}} \psi \geq-r \omega$. Soit $\left(a_{1}, a_{2}\right) \in X$ tel que $\psi\left(a_{1}, a_{2}\right)=0$. On veut estimer la $\mu$ mesure de $E:=(\psi<-t)$ pour $t \geq 0$. Posons

$$
F:=\left\{x_{2} \in X_{2}, \psi\left(a_{1}, x_{2}\right)<-t / 2\right\}
$$

et

$$
E_{x_{2}}:=\left\{x_{1} \in X_{1}, \psi\left(x_{1}, x_{2}\right)<-t\right\} .
$$

Définissons

$$
E^{\prime}:=\bigcup_{x_{2} \in X_{2} \backslash F}\left(E_{x_{2}} \times\left\{x_{2}\right\}\right)
$$

On a $E \subset \pi_{2}^{-1}(F) \cup E^{\prime}$.

Estimons la mesure de $\pi_{2}^{-1}(F)$. Si $\psi_{1}\left(x_{2}\right):=\psi\left(a_{1}, x_{2}\right)$, on a $\max _{X_{2}} \psi_{1}=$ $\psi_{1}\left(a_{2}\right)=0$. Posons $\psi_{2}:=\psi_{1}-\int \psi_{1} \mathrm{~d} \mu_{2}$. On a $\int \psi_{2} \mathrm{~d} \mu_{2}=0, \psi_{2} \geq \psi_{1}$ et $\mathrm{dd}^{\mathrm{c}} \psi_{2} \geq-r \omega_{2}$. Par définition de $\mathrm{R}_{1}\left(X_{2}, \omega_{2}, \mu_{2}\right)$, on a

$$
-\int \psi_{1} \mathrm{~d} \mu_{2}=\max _{X_{2}} \psi_{2} \leq r \mathrm{R}_{1}\left(X_{2}, \omega_{2}, \mu_{2}\right) \leq r \mathrm{R}
$$

$\operatorname{car} r\left(X_{2}, \omega_{2}\right) \geq 1$. D'où

$$
\begin{aligned}
\mu_{2}(F) & \leq \mu_{2}\left(\psi_{2} \leq r \mathrm{R}-t / 2\right)=\mu_{2}\left(r^{-1} \psi_{2} \leq \mathrm{R}-r^{-1} t / 2\right) \\
& \leq \Delta\left(X_{2}, \omega_{2}, \mu_{2}, \mathrm{R}-r^{-1} t / 2\right) \leq c \exp \left(\alpha \mathrm{R}-\alpha r^{-1} t / 2\right) \\
& =c \exp (\alpha \mathrm{R}) \exp \left(-\alpha r^{-1} t / 2\right) .
\end{aligned}
$$

Donc $\mu\left(\pi_{2}^{-1}(F)\right) \leq c \exp (\alpha \mathrm{R}) \exp \left(-\alpha r^{-1} t / 2\right)$.

Estimons la mesure de $E^{\prime}$. Pour $x_{2} \in X_{2} \backslash F$, posons $\psi_{3}\left(x_{1}\right):=\psi\left(x_{1}, x_{2}\right)$. On a $\psi_{3} \leq 0, \max _{X_{1}} \psi_{3} \geq \psi\left(a_{1}, x_{2}\right) \geq-t / 2$ et $\mathrm{dd}^{\mathrm{c}} \psi_{3} \geq-r \omega_{1}$. Posons $\psi_{4}:=$ $\psi_{3}-\int_{X_{1}} \psi_{3} \mathrm{~d} \mu_{1}$. On a

$$
-\int \psi_{3} \mathrm{~d} \mu_{1} \leq \max _{X_{2}} \psi_{4}+t / 2 \leq r \mathrm{R}_{1}\left(X_{2}, \omega_{2}, \mu_{2}\right)+t / 2 \leq r \mathrm{R}+t / 2 .
$$

et

$$
\begin{aligned}
\mu_{1}\left(E_{x_{2}}\right) & \leq \mu_{1}\left(\psi_{4} \leq r \mathrm{R}-t / 2\right) \leq c \exp \left(\alpha \mathrm{R}-\alpha r^{-1} t / 2\right) \\
& =c \exp (\alpha \mathrm{R}) \exp \left(-\alpha r^{-1} t / 2\right) .
\end{aligned}
$$

D'après le théorème de Fubini, on a $\mu\left(E^{\prime}\right) \leq c \exp (\alpha \mathrm{R}) \exp \left(-\alpha r^{-1} t / 2\right)$.

On déduit des estimations précédentes que pour tout $t \geq 0$

$$
\mu(\psi<-t) \leq 2 c \exp (\alpha \mathrm{R}) \exp \left(-\alpha r^{-1} t / 2\right) .
$$


C'est aussi vrai pour tout $t \in \mathbb{R}$ car $\psi \leq 0$. Si $\varphi$ est quasi-psh sur $X$ telle que $\operatorname{dd}^{\mathrm{c}} \varphi \geq$ $-\omega$ et $\int \varphi \mathrm{d} \mu=0$, on peut appliquer l'estimation précédente à $\psi:=\varphi-\max _{X} \varphi$. Comme $\psi \leq \varphi$ on a

$$
\mu(\varphi<-t) \leq 2 c \exp (\alpha \mathrm{R}) \exp \left(-\alpha r^{-1} t / 2\right) .
$$

Donc $\Delta(X, \omega, t) \leq 2 c \exp (\alpha \mathrm{R}) \exp \left(-\alpha r^{-1} t / 2\right)$.

Estimons $\mathrm{R}_{1}(X, \omega, \mu)$. Soit $\psi$ comme ci-dessus, il faut montrer que

$$
-\int \psi \mathrm{d} \mu \leq 2 r \mathrm{R}+2 r \alpha^{-1} \log c+4 \alpha^{-1} r
$$

On a pour tout $t_{0} \geq 0$

$$
\begin{aligned}
-\int \psi \mathrm{d} \mu & =\int_{0}^{+\infty} \mu(\psi \leq-t) \mathrm{d} t \\
& =\int_{0}^{t_{0}} \mu(\psi \leq-t) \mathrm{d} t+\int_{t_{0}}^{+\infty} \mu(\psi \leq-t) \mathrm{d} t \\
& \leq \int_{0}^{t_{0}} \mathrm{~d} t+\int_{t_{0}}^{+\infty} 2 c \exp (\alpha \mathrm{R}) \exp \left(-\alpha r^{-1} t / 2\right) \mathrm{d} t \\
& =t_{0}+4 c \exp (\alpha \mathrm{R}) \alpha^{-1} r \exp \left(-\alpha r^{-1} t_{0} / 2\right) .
\end{aligned}
$$

En prenant $t_{0}=2 r \mathrm{R}+2 r \alpha^{-1} \log c$, on obtient

$$
-\int \psi \mathrm{d} \mu \leq 2 r \mathrm{R}+2 r \alpha^{-1} \log c+4 \alpha^{-1} r .
$$

Considérons l'espace multiprojectif $\mathbb{P}^{k, l}:=\mathbb{P}^{k} \times \cdots \times \mathbb{P}^{k}$ ( $l$ fois). Notons $\pi_{i}$ la projection de $\mathbb{P}^{k, l}$ sur le $i$-ème facteur. Posons $\omega_{\mathrm{MP}}:=c_{k, l} \sum \pi_{i}^{*}\left(\omega_{\mathrm{FS}}\right)$ et soit $\Omega_{\mathrm{MP}}$ la mesure associée à la forme volume $\omega_{\mathrm{MP}}^{k l}$. La constante $c_{k, l}>0$ est choisie de sorte que $\Omega_{\mathrm{MP}}$ soit une mesure de probabilité. On a

$$
\left(c_{k, l}\right)^{-k l}=\left(\begin{array}{c}
k l \\
k
\end{array}\right)\left(\begin{array}{c}
k l-k \\
k
\end{array}\right) \ldots\left(\begin{array}{c}
2 k \\
k
\end{array}\right) \text {. }
$$

Observons que $c_{k, l} \leq 1$ et que si $l$ est fixé, $c_{k, l}$ est minorée par une constante positive indépendante de $k$. Pour cela, il suffit d'utiliser la formule d'équivalence de Stirling $n ! \simeq \sqrt{2 \pi n} n^{n} \exp (-n)$. Notons $m_{\mathrm{MP}}$ la mesure produit des $m_{\mathrm{FS}}$ sur $\mathbb{R} \mathbb{P}^{k, l}:=\mathbb{R} \mathbb{P}^{k} \times \cdots \times \mathbb{R}^{k}(l$ fois $)$.

Proposition A.9. Il existe des constantes $c>0, \alpha>0$ et $m>0$, qui ne dépendent que de l, telles que pour tout $k \geq 1$ on ait $\mathrm{R}_{i}\left(\mathbb{P}^{k, l}, \omega_{\mathrm{MP}}, \mu\right) \leq c(1+\log k), i=1,2,3$, $\Delta\left(\mathbb{P}^{k, l}, \omega_{\mathrm{MP}}, \mu, t\right) \leq c k^{m} \exp (-\alpha t)$ pour tout $t>0$ et pour $\mu=\Omega_{\mathrm{MP}}$ ou $\mu=m_{\mathrm{MP}}$.

Démonstration. Observons que la constante $r\left(\mathbb{P}^{k, l}, \omega_{\mathrm{MP}}\right)$ est majorée par $l$. Il suffit d'appliquer les propositions 2.4, A.3, A.5, A.7 et A.8. 
Vol. 81 (2006) Distribution des valeurs de transformations méromorphes et applications 257

\section{Références}

[1] H. Alexander, Projective capacity. In Recent developments in several complex variables, Ann. of Math. Studies 100, Princeton University Press, Princeton, N.J., 1981, 3-27. Zbl 0494.32001 MR 0627747

[2] J.-Y. Briend, J. Duval, Deux caractérisations de la mesure d'équilibre d'un endomorphisme de $\mathbb{P}^{k}(\mathbb{C})$. Publ. Math. Inst. Hautes Études Sci. 93 (2001), 145-159. Zbl 1010.37004 MR 1863737

[3] J.-P. Demailly, Regularization of closed positive currents and intersection theory. J. Algebraic Geom. 1 (1992), 361-409. Zbl 0777.32016 MR 1158622

[4] T.-C. Dinh, Distribution des préimages et des points périodiques d'une correspondance polynomiale. Bull. Soc. Math. France, à paraître.

[5] T.-C. Dinh, N. Sibony, Dynamique des applications d'allure polynomiale. J. Math. Pures Appl. 82 (2003), 367-423. Zbl 1033.37023 MR 1992375

[6] T.-C. Dinh, N. Sibony, Une borne supérieure pour l'entropie topologique d'une application rationnelle. Ann. of Math., à paraître.

[7] T.-C. Dinh, N. Sibony, Regularization of currents and entropy. Ann. Sci. École Norm. Sup. 37 (2004), 959-971. Zbl 02174959 MR 2119243

[8] D. Dolgopyat, On decay of correlations in Anosov flows. Ann. of Math. (2) 147 (1998), 357-390. Zbl 0911.58029 MR 1626749

[9] H. Federer, Geometric Measure Theory. Classics in Mathematics, Springer-Verlag, Berlin 1996. Zbl 0874.49001 MR 0257325

[10] J.-E. Fornæss, N. Sibony, Complex dynamics in higher dimension. In Complex potential theory (Montreal, PQ, 1993), NATO Adv. Sci. Inst. Ser. C Math. Phys. Sci. 439, Kluwer Academic Publ., Dordrecht 1994, 131-186. Zbl 0811.32019 MR 1332961

[11] B. Josefson, On the equivalence between locally polar and globally polar sets for plurisubharmonic functions on $\mathbb{C}^{n}$. Arkiv Mat. 16 (1978), 109-115. Zbl 0383.31003 MR 0590078

[12] V. Guedj, Ergodic properties of rational mappings with large topological degree. Ann. of Math., à paraître.

[13] L. Hörmander, The analysis of linear partial differential operators I. Grundlehren Math. Wiss. 256, Springer-Verlag, Berlin 1983. Zbl 0521.35001 MR 0717035

[14] L. Hörmander, An introduction to complex analysis in several variables. Third Edition, North-Holland Mathematical Library 7, North-Holland, Amsterdam 1990.Zbl 0685.32001 MR 1045639

[15] S. Kobayashi, Hyperbolic complex spaces. Grundlehren Math. Wiss. 318, Springer-Verlag, Berlin 1998. Zbl 0917.32019 MR 1635983

[16] K. Kodaira, D. C. Spencer, On deformations of complex analytic structures III. Ann. of Math. (2) 71 (1960), 43-76. Zbl 0128.16902 MR 0115189

[17] M. Ju. Lyubich, Entropy properties of rational endomorphisms of the Riemann sphere. Ergodic Theory Dynam. Systems 3 (1983), 351-385. Zbl 0537.58035 MR 0741393

[18] M. Méo, Image inverse d'un courant positif fermé par une application surjective. $C . R$. Acad. Sci. Paris Sér. I Math. 322 (1996), 1141-1144. Zbl 0858.32012 MR 1396655 
[19] S. Nonnenmacher, A. Voros, Chaotic eigenfunctions in phase space. J. Statist. Phys. 92 (1998), 431-518. Zbl 01357800 MR 1649013

[20] A. Russakovskii, B. Shiffman, Value distribution for sequences of rational mappings and complex dynamics. Indiana Univ. Math. J. 46 (1997), 897-932. Zbl 0901.58023 MR 1488341

[21] A. Russakovskii, M. Sodin, Equidistribution for sequences of polynomial mappings. Indiana Univ. Math. J. 44 (1995), 851-882. Zbl 0853.32006 MR 1375353

[22] B. Shiffman, S. Zelditch, Distribution of zeros of random and quantum chaotic sections of positive line bundles. Commun. Math. Phys. 200 (1999), 661-683. Zbl 0919.32020 MR 1675133

[23] N. Sibony, Dynamique des applications rationnelles de $\mathbb{P}^{k}$. In Dynamique et géométrie complexes (Lyon, 1997), Panor. Synthèses 8, Soc. Math. France, Paris, 1999, 97-185. Zbl 1020.37026 MR 1760844

[24] G. Tian, On a set of polarized Kähler metrics on algebraic manifolds. J. Differential Geom. 32 (1990), 99-130. Zbl 0706.53036 MR 1064867

[25] H. Triebel, Interpolation theory, function spaces, differential operators. North-Holland Mathematical Library 18, North-Holland Publishing Co., Amsterdam, New York 1978. Zbl 0387.46032 MR 0503903

[26] S. Zelditch, Szegö kernels and a theorem of Tian. Internat. Math. Res. Notices 6 (1998), 317-331. Zbl 0922.58082 MR 1616718

Received March 25, 2005; revised May 31, 2005

Tien-Cuong Dinh, Institut de Mathématique de Jussieu, Plateau 7D, Analyse Complexe, 175 rue du Chevaleret, 75013 Paris, France

E-mail:dinh@math.jussieu.fr

Nessim Sibony, Mathématique - Bâtiment 425, UMR 8628, Université Paris-Sud, 91405 Orsay, France

E-mail: nessim.sibony@math.u-psud.fr 